HLRZ 1998_25, WUB 98-21

\title{
Light and Strange Hadron Spectroscopy with Dynamical Wilson Fermions
}

\author{
N. Eicker, P. Lacock, K. Schilling, A. Spitz \\ HLRZ, Forschungszentrum Jülich, 52425 Jülich and \\ DESY, 22603 Hamburg, Germany \\ U. Glässner, S. Güsken, H. Hoeber, Th. Lippert, \\ Th. Struckmann, P. Ueberholz, J. Viehoff \\ Fachbereich Physik, Bergische Universität, Gesamthochschule Wuppertal \\ Gaußstraße 20, 42097 Wuppertal, Germany \\ G. Ritzenhöfer \\ Center for Theoretical Physics, Laboratory for Nuclear Science and Department of Physics, \\ Massachusetts Institute of Technology, Cambridge, MA 02139 \\ SESAM collaboration
}

Typeset using REVTEX 


\begin{abstract}
We present the final analysis of the light and strange hadron spectra from a full QCD lattice simulation with two degenerate dynamical sea quark flavours at $\beta=5.6$ on a $16^{3} \times 32$ lattice. Four sets of sea quark masses corresponding to the range $.69 \leq m_{\pi} / m_{\rho} \leq .83$ are investigated. For reference we also ran a quenched simulation at $\beta_{\text {eff }}=6.0$, which is the point of equal lattice spacing, $a_{\rho}^{-1}$.

In the light sector, we find the chiral extrapolation to physical $\mathrm{u}$ - and d- masses to present a major source of uncertainty, comparable to the expected size of unquenching effects. From linear and quadratic fits we can estimate the errors on the hadron masses made from light quarks to be on a $15 \%$ level prior to the continuum extrapolation. For the hadrons with strange valence quark content, the $N_{F}=2$ approximation to QCD appears not to cure the well-known failure of quenched QCD to reproduce the physical $K-K^{*}$ splitting.
\end{abstract}




\section{INTRODUCTION}

The nonperturbative computation of hadronic properties from Quantum Chromodynamics (QCD) presents a major challenge in the unraveling of quark flavourdynamics from hadronic experiments. The methods and tools of lattice gauge theory have been refined over the past two decades resulting in rather precise results (to the level of a few percent accuracy in the physical spectrum of light hadrons, i.e. after chiral and continuum extrapolations) within the quenched approximation [1]. High statistics quenched lattice studies on large lattice volumes revealed that the effects of dynamical fermions on spectrum and matrix elements appear to lie within a 10 to $20 \%$ range [2, 3].

The 'solution' of the full QCD binding problem with lattice methods, on the other hand, is still very much lagging behind. This is mainly due to the high cost in compute effort to encompass the fermionic determinant in the underlying stochastic sampling procedures. The simulation of large lattices in full QCD is definitely a task that requires the power of the upcoming teracomputers. Nevertheless, with the computing power of some several hundred of teraflops hours it is of considerable interest to tackle QCD vacuum polarization effects by looking - on intermediate volumes in the scaling regime - at quantities with inherent sea quark dependence such as the $\pi \mathrm{N} \sigma$-term, the $\eta^{\prime}$-mass, and the quark spin content of the nucleon.

A full QCD simulation with Wilson fermions is particularly expensive, as the fermionic operator in this case carries more degrees of freedom than in the staggered formulation, and its chiral extrapolation is more cumbersome as the chiral point fluctuates with the gauge field on a finite system. SESAM is a second generation simulation which is still exploratory, using Hybrid Monte Carlo (HMC) [4] at $\beta=5.6$ on $16^{3} \times 32$ lattices. For technical reasons, we work with two (degenerate) dynamical fermions, $N_{f}=2$. We devised several improvements in order to accelerate the computation of the fermionic force [5]. In this way, on the available APE100 hardware [6], we could achieve HMC histories of sufficient lengths for a safe estimate of autocorrelation times. This provides a sound basis for the error analysis.

In a full QCD computation there is no difference between sea quarks, which contribute to the fermion determinant, and valence quarks, which occur in the hadron operators that are employed to excite hadronic states from the QCD vacuum.

In our $N_{f}=2$ scenario, however, one is forced to introduce 'valence' quarks different from sea quarks, as soon as one wishes to deal with hadrons carrying strangeness. In a recent letter [7] devoted to the determination of the light and strange quark masses, we have therefore considered hadronic correlators on a set of three different sea quark masses, with valence quark content both equal and different to that of the underlying sea quark and presented a consistent approach to analyse such 'semiquenched' data.

In this paper we will extend that work from three to four different sea quark masses and present a detailed study of the light and strange hadron spectra. We shall identify sea and valence quarks in the light sector (of $u$ and d quarks) and resort to the semiquenched ansatz with respect to the strange quarks, as living in a sea of light quarks.

For reference, we perform a concomitant quenched simulation on equal lattice spac- 
ing and volume, at $\beta_{\text {eff }}=6.0 \mathrm{~W}$. While unquenching definitely leads to a considerable decrease of the light quark mass estimate [7], we find - within our errors - no visible sea quark effects both on the light and strange hadron masses.

\section{SIMULATION DETAILS}

\section{A. Hybrid Monte Carlo}

We have performed a large scale simulation of full QCD at $\beta=5.6$ with two degenerate flavors of dynamical Wilson fermions. We have generated lattices of extent $16^{3} \times 32$ at four different values of the sea-quark hopping parameter using the $\Phi$-version [8] of the HMC algorithm. The parameters used in the HMC update and the statistics for the complete runs on the 256 node APE100/Quadrics QH2 are given in table $\mathbb{1}$.

The CPU costs of the HMC are mostly due to the time consuming repeated solution of the linear system $M^{\dagger} M X=\Phi$ with $M$ being the Wilson fermion matrix. Throughout our simulation we employed the bi-conjugate gradient stabilized algorithm (BiCGStab) which has been demonstrated to be the most efficient Krylov sub-space solver for Wilson fermion inversions [5]. Using BiCGStab, we computed the linear system in a two step procedure

$$
M^{\dagger} Y=\Phi, \quad M X=Y
$$

In the first stage of the simulation, we preconditioned by use of the o/e decomposition of the Wilson fermion matrix $M \rightarrow M_{e}$ [9], $M_{e}=\mathbf{1}-\kappa^{2} D_{e o} D_{o e}$ referred to as $\mathrm{o} / \mathrm{e}$ in table $\mathbf{1}$. In a later stage of the simulation we switched from the thinned o/e representation $\operatorname{det}\left(M_{e}\right)$ to the full fermion determinant $\operatorname{det}(M)$ in order to employ the locally lexicographic SSOR preconditioner [10] which has been shown to offer up to a factor of 2 less computational costs than o/e preconditioning. In table 1 we refer to this part of the simulation as 'SSOR'.

As a third improvement of the molecular dynamics part within our HMC, we have implemented the chronological start vector guess [11]. The optimal depth of the extrapolation, $N_{C S G}$, has been determined empirically for each $\kappa_{\text {sea }}$ and with respect to the

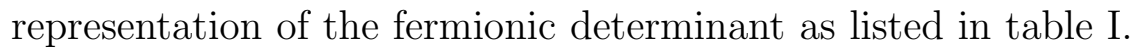

We have selected the time step size and the number of molecular dynamics steps, $N_{m d}$, to yield an acceptance rate of $>70 \%$ in the global Monte Carlo decision of HMC. With decreasing sea quark mass we can observe a variation of the acceptance rate from $85 \%$ to $73 \%$. We have varied the trajectory length $N_{m d}$ by numbers uniformly distributed in the range $\pm 2 \sqrt{N_{m d}}$ as recommended in Ref. [12] to avoid deadlocks in periodic orbits of phase space due to the presence of well defined Fourier modes.

\footnotetext{
${ }^{1}$ This value is at the onset of the (quenched) scaling regime.
} 
The chosen stopping accuracy, $R$, of the iterative solution of $M^{\dagger} M X=\Phi$ is the only source of systematic error of the HMC. We have defined the convergence criterion by $R=\frac{\|M X-\Phi\|}{\|X\|}=10^{-8}$ throughout our simulations, working at the level of APE's 32-bit precision. Beyond $R<10^{-7}$, the difference Hamiltonian $\Delta H$ for the global Monte Carlo decision, computed in double precision, does not vary significantly.

We proceeded adiabatically from large to small sea quark masses and, after thermalizing for more than 500 trajectories at each $\kappa_{\text {sea }}$, for each sea quark mass, we have generated 5000 trajectories. From these correlated samples we have chosen 200 decor-

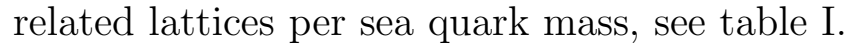

\section{B. Error estimates}

Since HMC is a Markov process, one is faced with the problem of autocorrelation of the generated series of trajectories. Of course one would like to aim at a decorrelated sample of configurations. However, since the generation of trajectories for full QCD is extremely costly we cannot afford to skip many trajectories as one can do in quenched simulations. In order to control the statistical quality of the measured signals we have to carefully study the autocorrelation of the Markov chain.

We paid attention to keep stable conditions for the HMC dynamics to evolve rather than re-tuning HMC parameters during production. This provides the setting for a reliable determination of the autocorrelation times related to various hadronic quantities.

For all four $\kappa_{\text {sea }}$ values, both exponential and integrated autocorrelation times of various gluonic and fermionic observables have been measured. The relevant quantity for the error determination is the integrated autocorrelation time $\tau_{\text {int }}$. We found $\tau_{\text {int }}$, which is observable-dependent, to be bound from above by $\tau_{\text {int }}^{\Lambda}$ of the smallest eigenvalue $\Lambda$ of the fermion matrix $\tau_{\text {int }}^{\Lambda}$ varies between 15 for $\kappa_{\text {sea }}=0.156$ and 30 for $\kappa_{\text {sea }}=0.1575$, however, the integrated autocorrelation times of most hadronic observables lie well below this limit. Therefore, we have decided to analyze every 25 th trajectory for spectrum and decay constants, after thermalization.

In order to account for possibly remaining correlations within our hadronic observables we have carried out a blocking investigation. For our smallest sea quark mass we show in fig.1 the errors of $m_{\pi}, m_{\rho}$ and $m_{N}$, as a function of the blocking size. At block size 4 to 6 we find the jackknife errors to run into plateaus. Accordingly, we shall use a block size of six throughout our analysis applying the bootstrap procedure. Errors (on the blocked data) are obtained from bootstrap samples with 250 entries each. A similar analysis of our quenched data shows no increase in error with the block size (quenched configurations are generated with an over-relaxed Cabbibo-Marinari heatbath update and are separated by 250 sweeps).

\footnotetext{
${ }^{2}$ In ref. [13] we shall present a detailed account of the underlying auto-correlation analysis, and we shall propose a scaling rule for the critical slowing down of the HMC.
} 
We remark that we have investigated the decorrelation efficiency of the HMC with respect to topology on the chosen samples[ [14]. It is gratifying that we could establish sufficient tunneling of the topological charge through the topological sectors for the four $\kappa_{\text {sea }}$ values investigated. For our smallest quark mass we determined an integrated autocorrelation time with respect to the topological charge of $\tau_{\text {int }} \approx 50$. Furthermore, we analyzed some hadronic quantities - which do not explicitly depend on topological effects - according to the topological charge content of the configurations. The result of this analysis is that no significant dependence on the topological sector was found.

\section{Hadronic observables}

At each of our four sea quark values we have investigated zero momentum two-point functions,

$$
C_{A B}(t)=\sum_{\vec{x}}\left\langle 0\left|\chi_{A}^{\dagger}(x) \chi_{B}(0)\right| 0\right\rangle
$$

with hadronic excitation operators $\chi$ as listed in table II. We combined light-quark propagators with hopping parameters equal and different to that of the underlying sea quark, thus providing ourselves with fifteen hadronic mass combinations at the two heaviest sea quarks and ten at the two lightest (see table III for the complete list).

We use the gauge-invariant Wuppertal-smearing procedure [15] to calculate "smeared-local" $(s l)$ and "smeared-smeared" $(s s)$ correlators. The smearing parameter is chosen to be $\alpha=4$, with $N=50$ iteration steps. In an attempt to further improve on our ground state projection we carried out an additional run with 100 smearing iterations at $\kappa_{\text {sea }}=0.1565$; although this rendered a somewhat faster drop into the ground state it did not alter our fit results. Plots with $\kappa_{\text {sea }}=0.1565$ are from our run with $N=100$.

Our analyses are based on global masses as extracted from single-exponential fits to the correlators:

$$
\begin{aligned}
& C(t)_{\mathrm{mes}}=A\left(e^{-m t}+e^{-m(T-t)}\right) \\
& C(t)_{\mathrm{bar}}=A e^{-m t},
\end{aligned}
$$

with $T=32$. As a cross check, we also determined effective local masses. For mesons they are computed iteratively from the implicit equation

$$
\frac{C_{A B}(t)}{C_{A B}(t+1)}=\frac{e^{-m_{\mathrm{eff}}(t) t}+e^{-m_{\mathrm{eff}}(t)(T-t)}}{e^{-m_{\mathrm{eff}}(t)(t+1)}+e^{-m_{\mathrm{eff}}(t)(T-t-1)}},
$$

\footnotetext{
${ }^{3}$ This investigation is a prerequisite for the investigation of quantities related to topology.
} 
while for baryons they are determined in the standard manner from the plateau of local masses:

$$
m_{\mathrm{eff}}(t)=\log \frac{\mathrm{C}_{\mathrm{AB}}(\mathrm{t})}{\mathrm{C}_{\mathrm{AB}}(\mathrm{t}+1)} .
$$

We use the smeared-smeared data to obtain both masses and amplitudes. The fit ranges are determined by keeping the upper limit fixed half-way across the lattice, while the lower cut in $t$ is varied in the interval $7-10^{\text {f }}$. The mass plateau range with the best $\chi^{2} /$ d.o.f-value is selected as fit interval.

Figure 2 illustrates the quality of our data by showing the different effective local masses in comparison to the global masses from correlated fits to the two-point functions, in the optimal fit ranges. We find that uncorrelated fits lead to consistent results.

For future reference our 'raw data' from these mass fits are collected in tables $\square$ to VIII. By inspection of these tables we retrieve $m_{\pi} / m_{\rho}$ ratios of $0.833(5), 0.809(15)$, $0.758(11)$ and $0.686(11)$ at $\kappa_{\text {sea }}=0.1560,0.1565,0.1570$ and 0.1575 respectively.

We determine the pseudoscalar and vector decay constants from the respective current matrix elements on the lattice:

$$
f_{\pi}=\frac{1}{m_{\pi}} Z_{A}\left\langle 0\left|A_{0}^{l}\right| \pi\right\rangle, \quad \frac{\epsilon_{\mu}}{f_{V}}=\frac{Z_{V}}{m_{V}^{2}}\left\langle 0\left|V_{\mu}^{l}\right| V\right\rangle .
$$

The matrix elements are extracted from a direct fit to the ratios

$$
\begin{aligned}
R_{P S} & =\frac{\left\langle 0\left|A_{0}^{l} A_{0}^{s}\right| 0\right\rangle}{\left\langle 0\left|A_{0}^{s} A_{0}^{s}\right| 0\right\rangle^{1 / 2}}=\left\langle 0\left|A_{0}^{l}\right| \pi\right\rangle e^{-m_{P S} \frac{T}{4}} \frac{1}{\sqrt{2 m_{P S}}} \cosh ^{\frac{1}{2}}\left(m_{P S}\left(t-\frac{T}{2}\right)\right) \\
R_{V} & =\frac{\left\langle 0\left|V^{l} V^{s}\right| 0\right\rangle}{\left\langle 0\left|V^{s} V^{s}\right| 0\right\rangle^{1 / 2}}=3^{-1 / 2}\left\langle 0\left|V_{\mu}^{l}\right| V\right\rangle e^{-m_{V} \frac{T}{4}} \frac{1}{\sqrt{2 m_{V}}} \cosh ^{\frac{1}{2}}\left(m_{V}\left(t-\frac{T}{2}\right)\right),
\end{aligned}
$$

where the superscripts $l$ and $s$ denote local and smeared operators, respectively. Note that in the second equation the operators $V V$ stand generically for $\sum_{k=1}^{3} V_{k} V_{k}$ while the operator $V$ on the r.h.s. denotes $\sum_{k=1}^{3} V_{k}$.

The masses in equations 6 and 7 are fixed to the values obtained from the mass fits (given in tables $\nabla$ through $\nabla \Pi 1$ ).

The 'raw data' for the lattice matrix elements $\left\langle 0\left|A_{0}^{l}\right| \pi\right\rangle$ and $3^{-1 / 2}\left\langle 0\left|V^{l}\right| V\right\rangle$ as well as $f_{\pi} / Z_{A}$ and $1 /\left(f_{V} Z_{V}\right)$ are collected in tables IX to XII.

The renormalization factors $Z_{A}$ and $Z_{V}$ are computed perturbatively, as explained in the Appendix.

\section{CHIRAL EXTRAPOLATIONS}

\footnotetext{
${ }^{4}$ The smeared-local data yield consistent results but correspond to smaller fit ranges in $t$.
} 


\section{A. The light sector}

We will first present our results for particles and decay constants containing nonstrange quarks only. It is obvious to identify the degenerate sea quarks in our simulation with the $u$ and $d$ quarks since, naively, we expect the lightest sea quarks to make the largest effect on the hadronic properties. In this scenario the light hadrons are determined from our raw data by a chiral extrapolation in quark mass. We call this setting "symmetric", since it involves data points with equal sea and valence quark masses only.

At this stage one should remember that full QCD vacuum configurations on different sea quark sectors are manifestly decorrelated. This has some bearing on the error analysis of hadron spectra, differently from the quenched situation where one normally determines entire hadron mass trajectories configuration wise, with ensuing point to point correlations. It will be interesting to trace the impact of this peculiarity on the accuracy of hadron masses and decay amplitudes under chiral extrapolation, in the full QCD situation.

\section{Masses and decay constants}

The pseudoscalar mass is used to extract the critical hopping parameter $\kappa_{\text {sea }}^{c}$ while the value of the light hopping parameter $\kappa_{\text {sea }}^{\text {light }}$ is set by the condition?:

$$
\frac{m_{\mathrm{PS}}\left(\kappa_{\mathrm{sea}}^{\text {light }}\right)}{m_{\mathrm{V}}\left(\kappa_{\mathrm{sea}}^{\text {light }}\right)}=\frac{M_{\pi}}{M_{\rho}}=0.1785 .
$$

The isospin symmetric bare light quark mass is given by:

$$
m^{\text {light }}=\frac{1}{2}\left(\frac{1}{\kappa_{\text {sea }}^{\text {light }}}-\frac{1}{\kappa_{\text {sea }}^{c}}\right) .
$$

Linear fits to our data for the pseudoscalar $\left(m_{\mathrm{PS}, \mathrm{ss}}^{2}\right)$ and vector masses $\left(m_{\mathrm{V}, \mathrm{ss}}\right)$ with $\kappa_{\text {sea }}=\kappa_{\text {val }}$ are shown in fig. 3. The resulting parameter values from the extrapolations are given in table XII], where we employ the following notations:

$$
\begin{aligned}
m_{P S}^{2} & =a+b \frac{1}{\kappa_{\text {sea }}} \\
m_{V} & =m^{c r i t}+c m_{\text {sea }}+e m_{\text {sea }}^{2} \quad \text { with } \quad m_{\text {sea }}=\frac{1}{2}\left(\frac{1}{\kappa_{\text {sea }}}-\frac{1}{\kappa_{\text {sea }}^{c}}\right) .
\end{aligned}
$$

We find the pseudoscalar data to be well described by the linear ansatz, the fit yielding the critical value of $\kappa_{\text {sea }}$ to be

\footnotetext{
${ }^{5}$ In this paper we use the convention that physical masses [16] are written in capital letters, while lattice masses are denoted by small letters.
} 


$$
\kappa_{\text {sea }}^{c}=0.158507_{-44}^{+41}
$$

with $\chi^{2} /$ d.o.f $=0.6$. For the vector particle, both linear and quadratic parametrizations yield acceptable fits, with $\chi^{2} /$ d.o.f $=0.75$ and $\chi^{2} /$ d.o.f $=0.27$, respectively. For the light hopping parameter, we find

$$
\left.\kappa_{\text {sea }}^{\text {light }}=0.158462_{-42}^{+41}(\text { linear }) \quad, \quad \kappa_{\text {sea }}^{\text {light }}=0.158471_{-45}^{+45} \text { (quadratic }\right) .
$$

In the light sector we quote the results from the linear ansatz, using the quadratic fit to estimate the systematic uncertainties. This gives the following value for the unrenormalized light quark mass

$$
m^{\text {light }}=0.000901(54)(184)
$$

the second error being the systematic uncertainty. Note that this value is consistent with our previous estimate, $m^{\text {light }}=0.00088(6)$ [7], obtained from simulations on three sea quark masses.

We can now predict the nucleon and $\Delta$ masses and the $\pi$ and $\rho$ decay constants by chiral extrapolation to the point $\kappa_{\text {sea }}^{\text {light }}$. The resulting fit parameters, in the notation of of eq. 11, are collected in table XII]. The extrapolations of the baryonic masses are visualized in fig. $\theta$. It turns out that their $m_{\text {sea }}$-dependence is by a factor 2 to 3 stronger than in the mesonic case, leading to a statistical error on the mass extrapolations for nucleon and $\Delta$ of 16 and $22 \%$, respectively. By comparing the deviations among linear and quadratic extrapolations, see table XIII, we might estimate a systematic error of 15 $\%$ and $24 \%$, respectively, which is covered by the statistical error, however. In order to put these numbers into perspective, one should be aware that we are extrapolating down from $m_{\pi} / m_{\rho}=.686$ on the basis of raw data, which carry statistical errors in the range of one to two \% (see tables $\mathrm{V}$ to VIII). Just for reference: in state-of-the-art quenched simulations [1] the Tsukuba group achieves statistical errors in the region of .5 to $1 \%$, in the range of $m_{\pi} / m_{\rho}$ down to a value of $0.4[3]$ !

For the decay constants we proceed similarly. The renormalized data are displayed in fig. 5; they favour the linear extrapolation (see also table XIII). Again, by comparing linear and quadratic results, we estimate our systematic uncertainties; they amount to 15 and $3 \%$ for $f_{\pi}$ and $f_{\rho}$, respectively.

\section{B. The strange sector}

So far we have used $M_{\pi}^{2}$ and $\frac{M_{\pi}}{M_{\rho}}$ to set the values of the hopping parameter values in the chiral limit and at the u quark mass. In the following we shall briefly describe our procedure to determine the value of the hopping parameter related to the strange quark mass [7].

Our simulations are based on two 'active', degenerate sea quarks, which we identify with the light quarks. The strange quark in this setting has to be treated as an effectively 
quenched quark that lives in the sea of the two physical light quarks. In order to account for this situation let us, for the sake of clarity, introduce the generic notation for various types of hadron masses appearing in the course of our calculations:

1. $m_{\mathrm{ss}}$ - both valence quarks are identical to the sea quark,

2. $m_{\mathrm{sv}}$ - one valence quark coincides with the sea quark,

3. $m_{\mathrm{vv}}$ - both valence quarks differ from the sea quark.

Note that the 'symmetric extrapolations' operate on the data set $m_{\mathrm{ss}}$ and suffice to determine both the critical and light hopping parameter values, as discussed in section IIIA.

Let us consider the pseudoscalar masses. In a linear parametrization, the three mass types can be written in terms of five slope parameters, $c$ to $d^{\prime \prime}$ :

$$
\begin{aligned}
& m_{\mathrm{PS}, \mathrm{ss}}^{2}=a^{\prime} m_{\mathrm{sea}} \\
& m_{\mathrm{PS}, \mathrm{sv}}^{2}=a^{\prime \prime} m_{\mathrm{sea}}+b^{\prime} m_{\mathrm{val}} \\
& m_{\mathrm{PS}, \mathrm{vv}}^{2}=a^{\prime \prime \prime} m_{\mathrm{sea}}+b^{\prime \prime}\left(m_{\mathrm{val} 1}+m_{\mathrm{val} 2}\right) .
\end{aligned}
$$

In the symmetric situation, $m_{\text {sea }}=m_{\text {val } 1}=m_{v a l 2}$, this mapping has to collapse to degeneracy on the l.h.s. which leads to constraints on the slopes. As a result one ends up with the simple form

$$
\left(\begin{array}{c}
m_{\mathrm{PS}, \mathrm{ss}}^{2} \\
m_{\mathrm{PS}, \mathrm{sv}}^{2} \\
m_{\mathrm{PS}, \mathrm{vv}}^{2}
\end{array}\right)=\left(\begin{array}{cc}
a^{\prime} & 0 \\
a^{\prime}-b^{\prime} & b^{\prime} \\
a^{\prime}-2 b^{\prime} & 2 b^{\prime}
\end{array}\right)\left(\begin{array}{c}
m_{\mathrm{sea}} \\
m_{\mathrm{val}}
\end{array}\right)
$$

which can be used for simultaneous fitting in $m_{\text {sea }}$ and $m_{\text {val }}$. In the spirit of our approach, we will identify the light quark mass with $m_{\text {sea }}$, while the strange quark mass is described by $m_{v a l}$. Note that through the degeneracy requirement, we are effectively left with two independent slopes only

Valence quark masses in eq. 15 are defined as:

$$
m_{\mathrm{val}}=\frac{1}{2}\left(\frac{1}{\kappa_{\mathrm{v}}}-\frac{1}{\kappa_{\mathrm{sea}}^{c}}\right) .
$$

In this setting, with the three types of hadron masses we are in the position to perform 'semiquenched extrapolations' where valence $\kappa$ values with $\kappa_{\text {val }} \neq \kappa_{\text {sea }}$ are admitted.

Within the linear ansatz, other hadronic quantities (like masses and decay constants) can be written in terms of $m_{\text {sea }}$ and $m_{\text {val }}$ in the generic form

\footnotetext{
${ }^{6}$ In our previous letter we used three independent such slopes [7].
} 


$$
\left(\begin{array}{c}
m_{\mathrm{ss}} \\
m_{\mathrm{sv}} \\
m_{\mathrm{vv}}
\end{array}\right)=m^{\prime \text { crit }}+\left(\begin{array}{cc}
c^{\prime} & 0 \\
c^{\prime}-d^{\prime} & d^{\prime} \\
c^{\prime}-2 d^{\prime} & 2 d^{\prime}
\end{array}\right)\left(\begin{array}{c}
m_{\mathrm{sea}} \\
m_{\mathrm{val}}
\end{array}\right) .
$$

We have performed semiquenched fits to eqs.15 and 17 using the subset of mesonic data with $\kappa_{\text {sea }}=\kappa_{\mathrm{val}_{1}}=\kappa_{\mathrm{val}_{2}}, \kappa_{\text {sea }}=\kappa_{\mathrm{val}_{1}} \neq \kappa_{\mathrm{val}_{2}}, \kappa_{\text {sea }} \neq \kappa_{\mathrm{val}_{1}}=\kappa_{\mathrm{val}_{2}}$, as described by the $\kappa$ combinations in table III. In order to ensure consistency with the above light sector analysis we have used the parameters from the symmetric fits as inputs, namely $a^{\prime}=2 b, \kappa_{\text {sea }}^{\text {light }}, \kappa_{\text {sea }}^{c}, m^{\prime c r i t}=m^{\text {crit }}, c^{\prime}=c$.

The results of such simultaneous fitting are listed in table $X \nabla$ and illustrated in the plots of fig. 6. All fits are characterized by reasonable $\chi^{2} /$ d.o.f. As we will discuss below, we have tested the stability of the procedure by relaxing the constraints.

It is obvious how to extend the analysis to nonlinear contributions in $m_{\text {sea }}$ and $m_{\text {val }}$ :

$$
m=m^{\prime \text { crit }}+\left(c^{\prime}-2 d^{\prime}\right) m_{m_{\text {sea }}}+2 d^{\prime} m_{\text {val }}+\left(e^{\prime}-f^{\prime}-g^{\prime}\right) m_{\text {sea }}^{2}+f^{\prime} m_{\text {sea }} m_{\text {val }}+g^{\prime} m_{\text {val }}^{2}
$$

The parameters from this nonlinear ansatz, with equal constraints from the above symmetric analysis, and with $e^{\prime}=e$, are also included in table XV. Note that the coefficients of $m_{\mathrm{sea}}^{2}$ and $m_{\mathrm{sea}} m_{\mathrm{val}}$ both come out to be negative, while the prefactor of $m_{\mathrm{val}}^{2}$ is positive but small.

Motivated by chiral perturbation theory and quenched QCD one might expect, instead of eq.17, a direct connection on the pseudoscalar mass, according to the form

$$
m=m^{\mathrm{crit}}+\tilde{b} m_{\mathrm{PS}}^{2},
$$

which amounts to restricting the parameters

$$
c^{\prime}=\tilde{b} a^{\prime}, d^{\prime}=\tilde{b} b^{\prime} .
$$

To check for the validity of this idea, we have entered our entire data set into a "scatterplot' with axes $m_{V}$ and $m_{P S}^{2}$. Fig. 7 reveals that the entries do not collapse to a single line but rather exhibit a clear pattern of sea quark mass dependence, thus ruling out the one-slope ansatz eq. [19].

\section{Determination of $\kappa^{\text {strange }}$}

There are three options to fix $\kappa^{\text {strange }}$ from the spectrum:

- from the $K^{*}$ mass by solving

$$
\frac{m_{\mathrm{V}, \mathrm{sv}}\left(\kappa_{\mathrm{sea}}^{\text {light }}, \kappa^{\mathrm{strange}}\right)}{m_{\mathrm{V}, \mathrm{ss}}\left(\kappa_{\mathrm{sea}}^{\text {light }}\right)}=\frac{M_{K^{*}}}{M_{\rho}}=1.16,
$$

where $\kappa_{\text {sea }}^{\text {light }}$ is given by eq. 13 ,

\footnotetext{
${ }^{7}$ Obviously this statement can be generalized to any dependence of type $m=f\left(m_{P S}\right)$.
} 
- or from the Kaon mass by matching

$$
\frac{m_{\mathrm{PS}, \mathrm{sv}}\left(\kappa_{\mathrm{sea}}^{\text {light }}, \kappa^{\text {strange }}\right)}{m_{\mathrm{PS}, \mathrm{ss}}\left(\kappa_{\mathrm{sea}}^{\text {light }}\right)}=\frac{M_{K}}{M_{\pi}}=3.61,
$$

- or from the $\Phi$ meson mass according to

$$
\frac{m_{V_{1}, \mathrm{vv}}\left(\kappa_{\mathrm{seat}}^{\text {light }}, \kappa^{\text {strange }}\right)}{m_{V_{2}, \mathrm{ss}}\left(\kappa_{\text {sea }}^{\text {light }}\right)}=\frac{M_{\phi}}{M_{\rho}}=1.326 .
$$

It is well known that quenched simulations with Wilson fermions fail to reproduce the size of the experimental hyperfine splitting among $K$ and $K^{*}$. According to the results of the CP-PACS collaboration [1], in the continuum limit and on large lattices, the value of $M_{K^{*}}\left(M_{\Phi}\right)$ turns out to deviate by $3 \%$ (5\%) from experiment when matching $\kappa^{\text {strange }}$ to $M_{K}$. On the other hand they find $M_{K^{*}}$ in accord with experiment when using $M_{\Phi}$ as input instead. The deviation is generally attributed to quenching errors.

In the context of the linear ansatz, the determination of $\kappa^{\text {strange }}$ from these alternative scale choices proceeds directly by explicit use of the fit parameters of table XV. Table $\mathrm{XVT}$ lists the resulting values. While the two vector conditions, eqs. 21 and 23, lead to consistent results, the $K$ meson mass condition asks for a considerably larger value of $\kappa^{\text {strange }}$.

With the numbers for $\kappa^{\text {strange }}$ from this table one can proceed to compute the meson masses in the strange sector, within the linear ansatz. The results are collected in table XVII. We find that, contrary to the expectation, the discrepancy between the lattice results and the experimental hyperfine splitting remains largely unaltered under unquenching. One might be tempted to blame the linear ansatz for this failure. However, as can be seen from table XV1, the spread in $\kappa^{\text {strange }}$ is by no means decreased under a quadratic extrapolation. We shall come back to this point when we discuss the $J$ parameter.

If one interprets the spread from the three strange quark mass settings as a systematic error, our 'best' value for the strange hopping parameter reads:

$$
\kappa^{\text {strange }}=0.15608(14)(46)
$$

which agrees with the mass ratios

$$
\frac{m_{K^{*}}}{m_{K}}=1.59(17) \quad \text { and } \quad \frac{m_{\Phi}}{m_{K}}=1.78(22) .
$$

The experimental mass ratios are $M_{K^{*}} / M_{K}=1.8$ and $M_{\Phi} / M_{K}=2.06$. The quoted value of $\kappa^{\text {strange }}$ implies a strange quark

$$
m \frac{\text { strange }}{\mathrm{MS}}(2 \mathrm{GeV})=151(30) \mathrm{MeV}
$$


to be compared to our previous value from the 3 sea quark analysis [7] $m_{\overline{\mathrm{MS}}}^{\text {strange }}(2 \mathrm{GeV})=$ 140(20)MeV.

The decay constants $f_{K}$ and $1 / f_{\phi}$ can be determined using the semi-quenched ansatz, eq.17, with $m$ replaced by $f_{P S}$ and $1 / f_{V}$ respectively. We compile the results in table

XVI1. It turns out that the conditions, eqs. 21, 22 and 23, lead to consistent answers, the spread of $3 \%$ being well covered by the statistical uncertainty.

\section{Stability of the semiquenched analysis}

By lifting the constraint one can convince oneself in two ways of the stability of the light sector, with respect to feedback from the strange sector:

(i) performing an unconstrained fit to eq. 15, ond finds (with $\chi^{2} /$ d.o.f $=22 / 29$ ) for the critical value of $\kappa_{\text {sea }}$

$$
\kappa_{\text {sea }}^{c}=0.158497_{-43}^{+47}
$$

which is nicely consistent with the result from the symmetric analysis, eq. 12.

(ii) An equally satisfying result is achieved with respect to $\kappa_{\text {sea }}^{\text {light }}$ the vector masses are reproduced with $\chi^{2} /$ d.o.f $=17 / 29$, and one obtains the value of the light hopping parameter as

$$
\kappa_{\text {sea }}^{\text {light }}=0.158451_{-41}^{+47}
$$

This number is also in good agreement with the outcome of the symmetric analysis, as given in eq. 13 .

\section{DISCUSSION}

\section{A. The J-parameter}

In the quenched scenario the dimensionless parameter

$$
J=M_{K^{*}} \frac{d M_{V}}{d M_{P S}^{2}}
$$

has been proposed as a suitable lattice observable to avoid chiral extrapolations altogether [17], on the level of the single mass (i.e. effective $\kappa$ ) dependence of the approximation. Assuming the vector and pseudoscalar trajectories to be linear one can estimate the slope within this expression from experimental mass ratio, such that

$$
J^{\mathrm{exp}} \simeq M_{K^{*}} \frac{M_{K^{*}}-M_{\rho}}{M_{K}^{2}-M_{\pi}^{2}}=0.48(2)
$$


The quenched lattice value for $J$ generally is of the order of .38, i.e. $25 \%$ below the empirical estimate. It has generally been surmised that this discrepancy provides evidence of a quenching error (see e.g. the review [1]).

Our approach to $N_{f}=2$ full QCD treats light and strange quarks on unequal footing, as we associate them with sea and valence quarks, respectively. Basically this induces a genuine two-parameter dependence of $m_{V}$ on the light and strange quark masses. Thus, in the $N_{f}=2$ theory, the notion of one effective hopping parameter is not appropriate and the lattice determination of $J$ does not eo ipso enjoy the merit of avoiding chiral extrapolation.

We have demonstrated in section III B 1 that our data does not confirm the single slope ansatz, eq.19. Thus, in order to avoid the problem of choosing an effective slope $\frac{d M_{V}}{d M_{P S}^{2}}$, we calculate the lattice value of $J$ directly from the experimental definition, i.e. we insert our lattice masses, c.f. tabs. XVII, XVII], on the r.h.s. of eq.30. We find

$$
J=0.33(3)
$$

if we define the strange quark mass by eq.22, and

$$
J=0.32(3)
$$

for the condition eq.21. Both values are well below the quenched results.

We compare this result with the outcome from an analysis restricted to the symmetric data. In this case an effective slope value $\tilde{b}=\frac{d M_{V}}{d M_{P S}^{2}}$ can be determined by a linear fit to $m_{V}\left(m_{P S}^{2}\right)$ on the symmetric data set. The result is $\tilde{b}_{\text {sym }}=1.07(6)$. Following ref. [17] we set the quark mass by the condition $m_{V}=1.8 m_{P S}$, which corresponds to the experimental $K^{*} / K$ mass ratio. This then produces the estimate $J=0.40(2)$, which is significantly above the result of our two-slope analysis, eqs. 31 or 32 . We disfavour this approach, however, since the (sea) quark mass, which fulfills the condition $m_{V}=1.8 m_{P S}$ on the symmetric line is purely effective and does not correspond to a sea of light $u$ and $d$ quarks.

A third possible way to estimate $J$ in the context of linear extrapolations is to apply the above procedure not to the symmetric line, but on each individual line of fixed sea quark mass, with subsequent extrapolation of $J$ in $\kappa_{\text {sea }}$. In this approach, however, one has to artificially impose the physical condition $m_{V}=1.8 m_{P S}$ on each one of the unphysical sea quark values. One can argue that a possible sea quark effect could be easily washed out by such unnaturally guided procedure. And indeed: we do not recover any appreciable dependence on the dynamical quark mass with this method. Moreover, the numbers, $J\left(\kappa_{\text {sea }}=0.1560\right)=0.34(5), J\left(\kappa_{\text {sea }}=0.1565\right)=$ $0.35(4), J\left(\kappa_{\text {sea }}=0.1570\right)=0.35(5), J\left(\kappa_{\text {sea }}=0.1575\right)=0.36(5)$ come out close to the quenched values.

Needless to say, the $J$-analysis does not provide us with independent information: obviously if we had succeeded in predicting the experimental Kaon and $K^{*}$ masses with a single value of $\kappa^{\text {strange }}$, the results for $J$, eqs. 31, 32, would agree exactly with $J^{\text {exp }}$. One might blame the linear ansatz for the failure of $J$ and $J^{\text {exp }}$ to coincide. In this 
sense this feature might be considered as an evidence for curvature in the vector particle trajectory. In order to explore this possibility, we have carrried out additional quadratic fits to the vector particle trajectory both on the symmetric and on the full data set. The results for the fit parameters can be found in tables $X I I]$ and $X \nabla$. The coefficients of the quadratic terms turn out to be negative, albeit zero within the errors. This then even lowers the value of $J$ !

As yet another alternative we have also used an ansatz with the next to linear order in the quark mass $\sim m^{3 / 2}$. Such a behaviour is expected by chiral perturbation theory [18]. However, we again find that the $J$ parameter decreases compared to the result of the linear ansatz.

\section{B. Consistency of scale determinations}

The lattice numbers for masses and decay constants can be translated into physical results once the lattice cutoff $a^{-1}$ at $\beta=5.6, N_{F}=2$ has been determined. This is done by matching the lattice number of one observable with its experimental counterpart. Obviously, within a complete numerical solution of QCD, the size of $a^{-1}$ should be independent of the choice of the particular observable selected to set the scale. Vice versa, a variation of the cutoff with the observable provides another measure for the systematic uncertainty of our lattice calculation.

Table XVIII exhibits the values of the cutoff as obtained by matching physical scales inside the light sector: the $\rho$ mass, the nucleon mass and the pion decay constant. Within statistical errors, all results appear consistent. The difference between $a_{N}^{-1}$ and $a_{f_{\pi}}^{-1}$ however reflects a systematic uncertainty of $\simeq 20 \%$, this being of course related to the the error from the chiral extrapolation, c.f. section IIIA.

The impact of the uncertainty due to the chiral extrapolation can also be demonstrated on $a_{\rho}^{-1}$ itself by making quadratic fits to the vector trajectory (see table XVIII). One observes a $10 \%$ change in $a_{\rho}^{-1}$ which goes along with an amplification of the error under the quadratic extrapolation from 4 to $15 \%$.

The physical predictions are collected in table XIX for the light sector, and in table $\mathrm{XX}$ for the masses of particles containing strange quarks. Here we used $a_{\rho}^{-1}$ both from linear and quadratic fits in order to test for the stability. It turns out that the admission of quadratic contributions in fitting the vector trajectory increases both baryon masses by 10 to $15 \%$ and does not reproduce the experimental $N-\Delta$ splitting. The uncertainty in the strange sector is clearly dominated by the mismatch of $\kappa^{\text {strange }}(K)$ and $\kappa^{\text {strange }}\left(K^{*}\right)$ which is connected with the failure to predict the experimental $K-K^{*}$ splitting. The physical results for $f_{K}$ and $1 / f_{\phi}$ are listed in table XXI. As we mentioned above, the uncertainty due to the choice of $\kappa^{\text {strange }}$ is covered by the statistical errors in this case.

The analogous quenched results $\left(\beta_{q}=6.0, a_{\rho}^{-1}=2.3[\mathrm{GeV}], 200\right.$ configurations of $16^{3} \times 32$ lattices) are contained in table XXII. Notice that the errors on the nucleon and $\Delta$ masses are smaller by a factor 2 to 4 .

In figs. 8 and 9 we present a compilation of the various quantities. We conclude 
that the data resists to reveal clear sea quark effect on these observables. In particular there remains the problem to account for the $N-\Delta$ mass splitting. It is unlikely that an increase in statistics would remedy the situation.

\section{SUMMARY AND CONCLUSIONS}

We have presented, on moderately sized lattices and at fixed $\beta$, a detailed analysis of the light and strange hadron spectra in full QCD. Both meet their particular difficulties: while the strange spectrum calculation is hampered by the technical requirement of $N_{F}=2$, the light baryonic sector faces the problem of considerable variation of hadron masses under chiral extrapolation.

We found that with these limitations we are not able to overcome the well-known shortcomings of quenched calculations, namely the underestimation of the $K-K^{*}$ and $N-\Delta$ spin splittings.

The experimental $J$-parameter cannot be explained in a linear scenario of vector trajectories and the admission of higher order terms does not help to improve on the situation. In view of this result, it would be highly desirable to make more realistic computations by including a third type of active sea quark.

The issue of the $N-\Delta$ splitting could be considerably clarified by closer approach to the chiral limit, on larger lattices. Work along these lines is in progress [19.

\section{Acknowledgements}

This work was done with support from the DFG grants Schi 257/1-4, 257/3-2, 257/33 and the DFG Graduiertenkolleg "Feldtheoretische und Numerische Methoden in der Statistischen und Elementarteilchenphysik". G.R. gratefully acknowledges the MaxKade Foundation for support. The HMC productions were run on the APE100 hardware at IfH Zeuthen and the Quadrics machine provided by DFG to the Schwerpunkt "Dynamische Fermionen", operated by the University of Bielefeld. Most of the hadron analysis was done on the CRAY T3E systems of ZAM at FZJ. We thank the support teams of these institutions. We thank G. Siegert for her contributions during the early stages of this work.

Last but not least, we are most grateful to the members of the INFN groups in at the Universities of Rome and Pisa, in particular R. Tripiccione and F. Rapuano for friendly cooperation; without the commitment of the APE team this work would not have been possible. 


\section{REFERENCES}

[1] T. Yoshié, Nucl.Phys. B (Proc.Suppl.) 63(1998)3.

[2] F. Butler et al, Nucl. Phys. B430 (1994)179.

[3] CP-PACS collaboration, S. Aoki et al., Nucl.Phys. (Proc.Suppl.) 60A (1998)14.

[4] S. Duane, A. Kennedy, B. Pendleton, and D. Roweth, Phys. Lett. B195 (1987)216.

[5] A. Frommer, V. Hannemann, Th. Lippert, B. Nöckel, and K. Schilling, Int. J. Mod. Phys. C5(1994)1073; A. Frommer, S. Güsken, Th. Lippert, B. Nöckel, and K. Schilling, Int. J. Mod. Phys. C6(1995)627.

[6] R. Tripiccione, Int.J.Mod.Phys. C4(1993)425.

[7] SESAM -Collaboration, N. Eicker et al, Phys. Lett.B407 (1997)290.

[8] S. Gottlieb, W. Liu, D. Toussaint, L. R. Renken, and R. L. Sugar, Phys. Rev. D 35 (1987) 2531.

[9] T. DeGrand and P. Rossi, Comp. Phys. Comm. 60 (1990) 211.

[10] S. Fischer et al., Comp. Phys. Comm. 98 (1996)20; Nucl. Phys. B (Proc. Suppl.)53 (1997)990.

[11] R. C. Brower, T. Ivanenko, A. R. Levi, K. N. Orginos, Nucl. Phys. B484 (1997) 353.

[12] P. Mackenzie, Phys. Lett. 226B (1989) 369.

[13] SESAM -Collaboration, Th. Lippert et al., Performance of the Hybrid Monte Carlo for QCD with Wilson fermions; , in preparation.

[14] B. Allés et al., IFUM 608-FT, IFUP-TH 10/98, HLRZ-98.6, HUP-EP-98/12, WUPTH 98-6, hep-lat/9803008.

[15] S. Güsken et al., Nucl. Phys. B(Proc. Suppl) 17(1990), 361.

[16] R.M. Barnett et al., Physical Review D54(1996)1 (Paticle Data Booklet). We use the following masses: $m_{\pi}=\frac{1}{2}\left(M_{\pi^{ \pm}}+M_{\pi^{0}}\right)=137.3 \mathrm{MeV} ; M_{\rho}=769 \mathrm{MeV} ; M_{\phi}=$ 1.019.4 GeV; $M_{K}=\frac{1}{2}\left(M_{K^{ \pm}}+M_{K^{0}}\right)=495.68 \mathrm{MeV} ; M_{K^{*}}=892 \mathrm{MeV}$.

[17] P. Lacock and C. Michael, Phys.Rev. D 52(1995)5213.

[18] E. Jenkins, A.V. Manohar, M.B. Wise, Phys. Rev. Lett. 75 (1995)2272.

[19] T $\chi$ L - Collaboration, L. Conti, L. Giusti, U. Glässner, S. Güsken, H. Hoeber, P. Lacock, Th. Lippert, G. Martinelli, F. Rapuano, G. Ritzenhöfer, K. Schilling, G. Siegert and A. Spitz; in preparation.

[20] G.P. Lepage and P.B. Mackenzie, Phys. Rev. D 48 (1993)2250. 


\section{APPENDIX A: RENORMALIZATION CONSTANTS}

We briefly present our method of choice for the extraction of the renormalization constants $Z_{A, V}$. We use the tadpole improved perturbation theory results from Lepage and Mackenzie [20]. The procedure is as follows:

- Use the plaquette values (see table XIV) to calculate the value of $\alpha_{V}\left(\frac{3.41}{a}\right)$ using:

$$
-\log \left\langle\frac{1}{3} \operatorname{Sp} \square\right\rangle=\frac{4 \pi}{3} \alpha_{V}(3.41 / a)\left(1-\left(1.191+0.025 n_{f}\right) \alpha_{V}\right) .
$$

- Convert to $\overline{M S}$ scheme using

$$
\frac{1}{\alpha_{\overline{M S}}(3.41 / a)}=\frac{1}{\alpha_{V}(3.41 / a)}+0.822 .
$$

- Run to a scale $\frac{1}{a}$.

- Use tadpole improved perturbation theory:

$$
Z_{A}=1-0.31 \alpha_{\overline{M S}}\left(\frac{1}{a}\right), \quad Z_{V}=1-0.82 \alpha \overline{M S}\left(\frac{1}{a}\right)
$$

- Neglecting the light quark dependence of the plaquette we find

$$
Z_{A}=0.93 \quad, \quad Z_{V}=0.82
$$

for $n_{f}=2$, and $Z_{A}=0.94, Z_{V}=0.83$ in the quenched case. We also need to rescale our quark fields:

$$
\sqrt{2 \kappa} \Psi \rightarrow \sqrt{1-3 \kappa / 4 \kappa_{c}} \Psi
$$

Matrix elements in the appendix are listed without the rescaling of the quark fields and before applying the renormalization constants. 
TABLES

TABLE I. Simulation parameters and characteristic numbers.

\begin{tabular}{|c|c|c|c|c|c|c|}
\hline \multicolumn{7}{|c|}{$\beta=5.6, N_{f}=2, V \times T=16^{3} \times 32$} \\
\hline \multirow{2}{*}{$\begin{array}{c}\kappa_{\text {sea }} \\
\text { Algorithm }\end{array}$} & \multirow{2}{*}{$\begin{array}{c}0.156 \\
\mathrm{o} / \mathrm{e}\end{array}$} & \multirow{2}{*}{$\begin{array}{l}0.1565 \\
\text { SSOR }\end{array}$} & \multicolumn{2}{|c|}{0.1570} & \multicolumn{2}{|c|}{0.1575} \\
\hline & & & $\mathrm{o} / \mathrm{e}$ & SSOR & $\mathrm{o} / \mathrm{e}$ & SSOR \\
\hline$T$ & 1 & 1 & 1 & 1 & 1 & 0.5 \\
\hline$N_{m d} \pm \sigma\left(N_{m d}\right)$ & $100 \pm 20$ & $100 \pm 20$ & $100 \pm 20$ & $100 \pm 20$ & $100 \pm 20$ & $71 \pm 12$ \\
\hline$N_{C S G}$ & 6 & 7 & 8 & 9 & 11 & 3 \\
\hline \# of iter. & $85(3)$ & $89(6)$ & $168(5)$ & $125(3)$ & $317(12)$ & $150(6)$ \\
\hline acc. $\operatorname{rate}[\%]$ & 85 & & 84 & 80 & 76 & 73 \\
\hline \# of traj. & 5000 & 5000 & 1500 & 3500 & 3000 & 2000 \\
\hline \# of confs. & 200 & 200 & & & 20 & \\
\hline$\kappa_{\text {val }}-\kappa_{\text {val }}$ comb. & 15 & 15 & & & 1 & \\
\hline
\end{tabular}

TABLE II. The operators studied.

\begin{tabular}{|ll|}
\hline \hline Mesons & $\chi_{A}^{\dagger}(x) \chi_{A}(0)$ \\
\hline Pseudoscalar: & $\chi_{P S}(x)=P_{5}=\bar{q}^{\prime}(x) \gamma^{5} q(x)$ \\
Vector: & $\chi_{V}^{\mu}(x)=V^{\mu}=\bar{q}^{\prime}(x) \gamma^{\mu} q(x)$ \\
Scalar: & $\chi_{S c}(x)=\bar{q}^{\prime}(x) q(x)$ \\
Axial-vector: & $\chi_{A x}(x)=A^{\mu}=\bar{q}^{\prime}(x) \gamma_{5} \gamma^{\mu} q(x)$ \\
\hline \hline Baryons & $\chi_{A}^{\dagger}(x) \chi_{A}(0)$ \\
\hline Nucleon: & $\chi_{N}(x)=\epsilon_{a b c}\left(q_{a} C \gamma_{5} q_{b}\right) q_{c}$ \\
$\Delta:$ & $\chi_{\Delta}^{\mu}(x)=\epsilon_{a b c}\left(q_{a} C \gamma^{\mu} q_{b}\right) q_{c}$ \\
\hline \hline Decay Constants & $\chi_{A}^{\dagger}(x), \chi_{B}(0)$ \\
\hline Pseudoscalar: & $\left(A_{0}^{\dagger}, P_{5}\right),\left(P_{5}^{\dagger}, A_{0}\right),\left(A_{0}^{\dagger}, A_{0}\right)$ \\
Vector: & $\left(V_{i}^{\dagger}, V_{i}\right)$ \\
\hline \hline
\end{tabular}


TABLE III. The run parameters for $\kappa_{v a l}$

\begin{tabular}{|c|c|}
\hline \hline$\kappa_{\text {sea }}$ & $\left\{\kappa_{\text {val }}\right\}$ \\
\hline 0.156 & $\{0.156,0.157,0.1575,0.158,0.1585\}$ \\
\hline 0.1565 & $\{0.156,0.1565,0.157,0.1575,0.158\}$ \\
\hline 0.157 & $\{0.1555,0,1565,0.157,0.1575\}$ \\
\hline 0.1575 & $\{0.1555,0,1565,0.157,0.1575\}$ \\
\hline \hline
\end{tabular}

TABLE IV. Integrated autocorrelation times $\tau_{\text {int }}$ for pseudoscalar, vector and nucleon for smeared-local and smeared-smeared correlators (numbers are in units of HMC time).

\begin{tabular}{|rcc|rr|rr|rr|r|}
\hline \hline$\beta$ & $\kappa$ & $V$ & \multicolumn{2}{|c}{$\tau_{\text {int }}\left(M_{P S}\right)$} & \multicolumn{2}{c}{$\tau_{\text {int }}\left(M_{V}\right)$} & \multicolumn{2}{c}{$\tau_{\text {int }}\left(M_{N}\right)$} & \multicolumn{3}{c|}{$B$} \\
\hline & & & sl & ss & sl & ss & sl & ss & \\
\hline \hline 5.6 & 0.1560 & $16^{3} \times 32$ & $22(9)$ & $<25$ & $<25$ & $<25$ & $<25$ & $<25$ & 6 \\
\hline 5.6 & 0.1570 & $16^{3} \times 32$ & $19(6)$ & $17(5)$ & $<25$ & $<25$ & $<25$ & $<25$ & 6 \\
\hline 5.6 & 0.1575 & $16^{3} \times 32$ & $44(20)$ & $33(22)$ & $<25$ & $<25$ & $37(20)$ & $32(24)$ & 7 \\
\hline \hline
\end{tabular}


TABLE V. Lattice results for the masses of Pion, Rho, Nucleon and Delta at $\kappa_{\text {sea }}=0.156$. For all fits we find $0.4 \leq \chi^{2} /$ d.o.f $\leq 1$.

\begin{tabular}{|c|c|c|c|c|}
\hline \multicolumn{5}{|c|}{$\kappa_{\text {sea }}=0.156$} \\
\hline \multicolumn{5}{|c|}{ nconfigs $=198$, nboot $=200$, correlated } \\
\hline$\left(t_{\min }, t_{\max }\right)$ & $(9,15)$ & $(9,15)$ & $(9,14)$ & $(8,14)$ \\
\hline$\kappa_{1}-\kappa_{2}$ & $m_{\pi}$ & $m_{\rho}$ & $m_{N}$ & $m_{\Delta}$ \\
\hline $0.1585-0.1585$ & 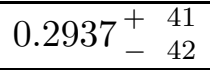 & $\begin{array}{ll}0.4422_{-}^{+} & 64 \\
\end{array}$ & $\begin{array}{ll}0.676_{-}^{+} 19 \\
\end{array}$ & $\begin{array}{l}0.788_{-}^{+} \\
\end{array}$ \\
\hline $0.1585-0.1580$ & $\begin{array}{l}0.3111_{-}^{+} 43 \\
\end{array}$ & $\begin{array}{ll}0.4507_{-}^{+} & 62 \\
& 67\end{array}$ & $\begin{array}{l}0.689_{-}^{+} 17 \\
\end{array}$ & $\begin{array}{l}0.796_{-}^{+} 18 \\
\end{array}$ \\
\hline $0.1580-0.1580$ & 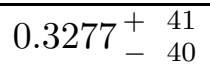 & $\begin{array}{l}0.4591_{-}^{+} 58 \\
\end{array}$ & $\begin{array}{l}0.711_{-}^{+} 16 \\
\end{array}$ & $\begin{array}{l}0.809_{-}^{+} 17 \\
\end{array}$ \\
\hline $0.1585-0.1575$ & $\begin{array}{ll}0.3279_{-}^{+} & 41 \\
\end{array}$ & $\begin{array}{l}0.4597_{-}^{+} \\
58\end{array}$ & $\begin{array}{l}0.702_{-}^{+} 15 \\
\end{array}$ & $\begin{array}{ll}0.804_{-}^{+} & 18 \\
0.17\end{array}$ \\
\hline $0.1580-0.1575$ & $\begin{array}{l}0.3438_{-}^{+39} \\
38\end{array}$ & $\begin{array}{l}0.4681_{-}^{+} 60 \\
\end{array}$ & $\begin{array}{l}0.724_{-}^{+} 15 \\
\end{array}$ & $\begin{array}{l}0.817_{-}^{+} \\
-16\end{array}$ \\
\hline $0.1585-0.1570$ & $\begin{array}{ll}0.3443_{-}^{+} & 39 \\
\end{array}$ & $\begin{array}{l}0.4691_{-}^{+} 58 \\
\end{array}$ & $\begin{array}{l}0.715_{-}^{+} 15 \\
\end{array}$ & $\begin{array}{l}0.812_{-}^{+} 16 \\
\end{array}$ \\
\hline $0.1575-0.1575$ & $0.3594_{-}^{+36}$ & $0.4771_{-}^{+} 59$ & $\begin{array}{ll}0.747_{-}^{+} 14 \\
\end{array}$ & $\begin{array}{l}0.832_{-}^{+} 16 \\
\end{array}$ \\
\hline $0.1580-0.1570$ & 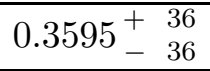 & $\begin{array}{l}0.4775_{-}^{+} 58 \\
\end{array}$ & $\begin{array}{l}0.737_{-}^{+} 14 \\
\end{array}$ & $\begin{array}{l}0.825_{-}^{+} \\
0.16\end{array}$ \\
\hline $0.1575-0.1570$ & $0.3745_{-}^{+34}$ & $0.4865_{-}^{+} 59$ & $\begin{array}{l}0.760_{-}^{+} 13 \\
\end{array}$ & $\begin{array}{l}0.840_{-}^{+} \\
15\end{array}$ \\
\hline $0.1585-0.1560$ & $0.3756_{-}^{+36}$ & $\begin{array}{l}0.4884_{-}^{+} 55 \\
\end{array}$ & $\begin{array}{l}0.742_{-}^{+} 13 \\
\end{array}$ & $\begin{array}{l}0.830_{-}^{+} 16 \\
\end{array}$ \\
\hline $0.1570-0.1570$ & $0.3892_{-}^{+33}$ & $\begin{array}{ll}0.4958_{-}^{+} & 60 \\
\end{array}$ & $\begin{array}{l}0.783_{-}^{+} 13 \\
\end{array}$ & $\begin{array}{l}0.857_{-}^{+} \\
-14\end{array}$ \\
\hline $0.1580-0.1560$ & $\begin{array}{l}0.3899_{-}^{+} 33 \\
\end{array}$ & $\begin{array}{l}0.4969_{-}^{+} 57 \\
\end{array}$ & $\begin{array}{l}0.764_{-}^{+} 13 \\
\end{array}$ & $\begin{array}{l}0.843_{-}^{+} 15 \\
\end{array}$ \\
\hline $0.1575-0.1560$ & $\begin{array}{l}0.4040_{-}^{+} 33 \\
\end{array}$ & $\begin{array}{l}0.5059_{-}^{+} 56 \\
\end{array}$ & $\begin{array}{ll}0.786_{-}^{+} 12 \\
\end{array}$ & $\begin{array}{l}0.858_{-}^{+} 13 \\
\end{array}$ \\
\hline $0.1570-0.1560$ & $\begin{array}{l}0.4179_{-}^{+} 35 \\
\end{array}$ & $0.5152_{-}^{+} 51$ & $0.808_{-}^{+} 11$ & $\begin{array}{l}0.875_{-}^{+} 14 \\
\end{array}$ \\
\hline $0.1560-0.1560$ & $\begin{array}{l}0.4452_{-}^{+} 32 \\
\end{array}$ & $\begin{array}{l}0.5345_{-}^{+} 54 \\
\end{array}$ & $\begin{array}{l}0.852_{-}^{+} 10 \\
\end{array}$ & $\begin{array}{l}0.910_{-}^{+} 12 \\
\end{array}$ \\
\hline
\end{tabular}


TABLE VI. Lattice results for the masses of Pion, Rho, Nucleon and Delta at $\kappa_{\text {sea }}=0.1565$. We find $1.5 \leq \chi^{2} /$ d.o.f $\leq 3$ for fits to $\pi$ and $\rho$, and $0.5 \leq \chi^{2} /$ d.o.f $\leq 1.5$ for fits to nucleon and $\Delta$.

\begin{tabular}{|c|c|c|c|c|}
\hline \multicolumn{5}{|c|}{$\kappa_{\text {sea }}=0.1565$} \\
\hline \multicolumn{5}{|c|}{ nconfigs $=198$, nboot $=200$, correlated } \\
\hline$\left(t_{\min }, t_{\max }\right)$ & $(9,15)$ & $(9,15)$ & $(9,14)$ & $(8,14)$ \\
\hline$\kappa_{1}-\kappa_{2}$ & $m_{\pi}$ & $m_{\rho}$ & $m_{N}$ & $m_{\Delta}$ \\
\hline $0.1580-0.1580$ & $\begin{array}{ll}0.3092_{-}^{+} & 61 \\
\end{array}$ & $\begin{array}{l}0.4408_{-}^{+} 91 \\
\end{array}$ & $\begin{array}{l}0.656_{-}^{+} 19 \\
\end{array}$ & $\begin{array}{l}0.704_{-}^{+} 37 \\
\end{array}$ \\
\hline $0.1580-0.1575$ & $\begin{array}{l}0.3257_{-}^{+} 59 \\
\end{array}$ & $\begin{array}{l}0.4496_{-81}^{+82} \\
\end{array}$ & $\begin{array}{l}0.673_{-}^{+20} \\
\end{array}$ & $\begin{array}{l}0.721_{-}^{+} 35 \\
\end{array}$ \\
\hline $0.1575-0.1575$ & $\begin{array}{l}0.3416_{-}^{+} 58 \\
\end{array}$ & $\begin{array}{l}0.4582_{-}^{+84} \\
\end{array}$ & $\begin{array}{l}0.701_{-}^{+} 18 \\
\end{array}$ & $\begin{array}{l}0.748_{-}^{+} 33 \\
0\end{array}$ \\
\hline $0.1580-0.1570$ & $\begin{array}{l}0.3418_{-}^{+58} \\
\end{array}$ & $\begin{array}{l}0.4588_{-}^{+85} \\
\end{array}$ & $\begin{array}{l}0.688_{-}^{+} 19 \\
\end{array}$ & $\begin{array}{l}0.736_{-}^{+34} \\
\end{array}$ \\
\hline $0.1575-0.1570$ & $\begin{array}{l}0.3572_{-}^{+54} \\
\end{array}$ & $\begin{array}{l}0.4675_{-}^{+84} \\
\end{array}$ & $\begin{array}{l}0.716_{-}^{+} 17 \\
\end{array}$ & $\begin{array}{l}0.763_{-}^{+} 31 \\
26\end{array}$ \\
\hline $0.1580-0.1565$ & $\begin{array}{l}0.3575_{-}^{+} 54 \\
\end{array}$ & $\begin{array}{l}0.4683_{-}^{+86} \\
\end{array}$ & $\begin{array}{l}0.702_{-}^{+} 18 \\
\end{array}$ & $\begin{array}{l}0.750_{-}^{+} 34 \\
\end{array}$ \\
\hline $0.1570-0.1570$ & $\begin{array}{l}0.3723_{-}^{+} 54 \\
\end{array}$ & $\begin{array}{l}0.4770_{-}^{+} \\
\end{array}$ & $\begin{array}{l}0.741_{-}^{+} 17 \\
\end{array}$ & $\begin{array}{l}0.789_{-}^{+} 26 \\
\end{array}$ \\
\hline $0.1575-0.1565$ & $\begin{array}{l}0.3724_{-}^{+} 53 \\
\end{array}$ & $\begin{array}{l}0.4772_{-}^{+} 83 \\
\end{array}$ & $\begin{array}{l}0.730_{-}^{+} 17 \\
\end{array}$ & $\begin{array}{l}0.776_{-}^{+} 28 \\
\end{array}$ \\
\hline $0.1570-0.1565$ & $\begin{array}{l}0.3871_{-}^{+} 53 \\
\end{array}$ & $\begin{array}{l}0.4868_{-}^{+} 74 \\
\end{array}$ & $\begin{array}{l}0.755_{-}^{+} 17 \\
\end{array}$ & $\begin{array}{l}0.802_{-}^{+} 26 \\
\end{array}$ \\
\hline $0.1580-0.1560$ & $\begin{array}{l}0.3728_{-}^{+} 54 \\
\end{array}$ & $\begin{array}{l}0.4782_{-}^{+} 87 \\
\end{array}$ & $\begin{array}{l}0.716_{-}^{+} 18 \\
0\end{array}$ & $\begin{array}{l}0.763_{-}^{+} 32 \\
\end{array}$ \\
\hline $0.1565-0.1565$ & $\begin{array}{l}0.4016_{-}^{+52} \\
\end{array}$ & $\begin{array}{l}0.4966_{-}^{+} 69 \\
\end{array}$ & $\begin{array}{l}0.778_{-}^{+} 16 \\
0\end{array}$ & $\begin{array}{l}0.827_{-22}^{+23} \\
\end{array}$ \\
\hline $0.1575-0.1560$ & $\begin{array}{l}0.3873_{-}^{+} 54 \\
\end{array}$ & $\begin{array}{l}0.4872_{-}^{+} 79 \\
\end{array}$ & $\begin{array}{l}0.744_{-}^{+} 18 \\
\end{array}$ & $\begin{array}{l}0.789_{-}^{+} 26 \\
\end{array}$ \\
\hline $0.1570-0.1560$ & $\begin{array}{l}0.4017_{-}^{+} 52 \\
\end{array}$ & $\begin{array}{l}0.4968_{-}^{+} \\
\end{array}$ & $\begin{array}{l}0.768_{-}^{+} 17 \\
\end{array}$ & $\begin{array}{l}0.815_{-}^{+24} \\
\end{array}$ \\
\hline $0.1565-0.1560$ & $\begin{array}{l}0.4157_{-}^{+} 51 \\
\end{array}$ & $\begin{array}{l}0.5067_{-}^{+68} \\
66\end{array}$ & $\begin{array}{l}0.792_{-}^{+} 16 \\
0\end{array}$ & $\begin{array}{l}0.839_{-}^{+23} \\
\end{array}$ \\
\hline $0.1560-0.1560$ & $\begin{array}{l}0.4295_{-}^{+} 40 \\
\end{array}$ & $\begin{array}{l}0.5168_{-}^{+63} \\
\end{array}$ & $\begin{array}{l}0.814_{-}^{+} 15 \\
0\end{array}$ & $\begin{array}{l}0.862_{-}^{+} 20 \\
0\end{array}$ \\
\hline
\end{tabular}


TABLE VII. Lattice results for the masses of Pion, Rho, Nucleon and Delta at $\kappa_{\text {sea }}=0.157$ We find $1.5 \leq \chi^{2} /$ d.o.f $\leq 3$ for fits to $\rho$ and $\Delta$, and $0.5 \leq \chi^{2} /$ d.o.f $\leq 1.5$ for fits to $\pi$ and nucleon.

\begin{tabular}{|c|c|c|c|c|}
\hline \multicolumn{5}{|c|}{$\kappa_{\text {sea }}=0.157$} \\
\hline \multicolumn{5}{|c|}{ nconfigs $=198$, nboot $=200$, correlated } \\
\hline$\left(t_{\min }, t_{\max }\right)$ & $(8,15)$ & $(8,15)$ & $(9,14)$ & $(9,14)$ \\
\hline$\kappa_{1}-\kappa_{2}$ & $m_{\pi}$ & $m_{\rho}$ & $m_{N}$ & $m_{\Delta}$ \\
\hline $0.1575-0.1575$ & 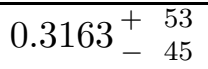 & $\begin{array}{r}0.4397_{-}^{+} \\
\end{array}$ & $\begin{array}{l}0.695_{-}^{+} \\
\end{array}$ & $\begin{array}{l}0.774_{-}^{+} 13 \\
\end{array}$ \\
\hline $0.1575-0.1570$ & $\begin{array}{l}0.3328_{-}^{+} 50 \\
02\end{array}$ & $\begin{array}{l}0.4499_{-}^{+} \\
\end{array}$ & $0.708_{-}^{+} 15$ & $\begin{array}{l}0.786_{-}^{+} 12 \\
\end{array}$ \\
\hline $0.1570-0.1570$ & $\begin{array}{l}0.3486_{-}^{+} 49 \\
0\end{array}$ & $\begin{array}{l}0.4600_{-}^{+} 70 \\
\end{array}$ & $\begin{array}{l}0.732_{-}^{+} 14 \\
\end{array}$ & $\begin{array}{l}0.805_{-}^{+} 13 \\
0\end{array}$ \\
\hline $0.1575-0.1565$ & 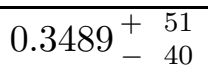 & $\begin{array}{l}0.4603_{-}^{+} 72 \\
\end{array}$ & $\begin{array}{l}0.722_{-}^{+} 15 \\
\end{array}$ & $\begin{array}{l}0.798_{-}^{+} 12 \\
\end{array}$ \\
\hline $0.1570-0.1565$ & $\begin{array}{l}0.3641_{-}^{+} 47 \\
0\end{array}$ & $\begin{array}{l}0.4703_{-}^{+} 67 \\
\end{array}$ & $0.746_{-}^{+} 13$ & $\begin{array}{l}0.816_{-}^{+} 14 \\
\end{array}$ \\
\hline $0.1565-0.1565$ & $\begin{array}{l}0.3790_{-}^{+} \\
\end{array}$ & $\begin{array}{l}0.4805_{-}^{+} \\
64\end{array}$ & $\begin{array}{l}0.769^{+} 12 \\
-12\end{array}$ & $\begin{array}{l}0.835_{-}^{+} 14 \\
\end{array}$ \\
\hline $0.1575-0.1555$ & $\begin{array}{c}0.3797_{-}^{+} \\
\end{array}$ & $\begin{array}{l}0.4812_{-}^{+} \\
06\end{array}$ & $\begin{array}{l}0.750_{-}^{+} 14 \\
\end{array}$ & $\begin{array}{c}0.819^{+} 14 \\
-14\end{array}$ \\
\hline $0.1570-0.1555$ & $\begin{array}{c}0.3939_{-}^{+} \\
\end{array}$ & 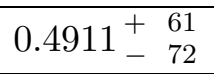 & $0.774_{-}^{+} 13$ & $\begin{array}{c}0.837_{-}^{+} 14 \\
\end{array}$ \\
\hline $0.1565-0.1555$ & $\begin{array}{c}0.4080_{-}^{+} \\
\end{array}$ & $\begin{array}{c}0.5010_{-}^{+} \\
\end{array}$ & $0.797_{-}^{+} 12$ & $\begin{array}{l}0.856_{-}^{+} 13 \\
\end{array}$ \\
\hline $0.1555-0.1555$ & $\begin{array}{c}0.4354_{-}^{+} \\
\end{array}$ & $\begin{array}{c}0.5211_{-}^{+} \\
-61\end{array}$ & $\begin{array}{l}0.842_{-}^{+} 10 \\
0\end{array}$ & $\begin{array}{l}0.895_{-}^{+} 14 \\
\end{array}$ \\
\hline
\end{tabular}


TABLE VIII. Lattice results for the masses of Pion, Rho, Nucleon and Delta at $\kappa_{\text {sea }}=0.1575$. For all fits we find $0.5 \leq \chi^{2} /$ d.o.f $\leq 1.5$.

\begin{tabular}{|c|c|c|c|c|}
\hline \multicolumn{5}{|c|}{$\kappa_{\text {sea }}=0.1575$} \\
\hline \multicolumn{5}{|c|}{ nconfigs $=198$, nboot $=200$, correlated } \\
\hline$\left(t_{\min }, t_{\max }\right)$ & $(7,15)$ & $(10,15)$ & $(8,14)$ & $(8,14)$ \\
\hline$\kappa_{1}-\kappa_{2}$ & $m_{\pi}$ & $m_{\rho}$ & $m_{N}$ & $m_{\Delta}$ \\
\hline $0.1575-0.1575$ & $\begin{array}{ll}0.2803_{-}^{+} & 45 \\
\end{array}$ & $\begin{array}{l}0.4087_{-}^{+} \\
\end{array}$ & $\begin{array}{ll}0.633_{-}^{+} 12 \\
\end{array}$ & $\begin{array}{l}0.695_{-}^{+} 16 \\
\end{array}$ \\
\hline $0.1575-0.1570$ & $\begin{array}{ll}0.2986_{-}^{+} & 42 \\
\end{array}$ & $\begin{array}{l}0.4178_{-}^{+} 56 \\
\end{array}$ & $0.648_{-}^{+} 10$ & $\begin{array}{l}0.707_{-}^{+} 15 \\
\end{array}$ \\
\hline $0.1570-0.1570$ & $\begin{array}{l}0.3159_{-}^{+} 37 \\
\end{array}$ & $\begin{array}{ll}0.4272_{-}^{+} & 52 \\
\end{array}$ & $\begin{array}{ll}0.673_{-}^{+} & 8 \\
\end{array}$ & $\begin{array}{c}0.730_{-}^{+} 14 \\
\end{array}$ \\
\hline $0.1575-0.1565$ & $\begin{array}{ll}0.3160_{-}^{+} & 37 \\
\end{array}$ & $\begin{array}{l}0.4275_{-}^{+54} \\
51\end{array}$ & $\begin{array}{rr}0.662_{-}^{+} & 9 \\
\end{array}$ & $\begin{array}{l}0.719_{-}^{+} 15 \\
\end{array}$ \\
\hline $0.1570-0.1565$ & $\begin{array}{ll}0.3325_{-}^{+} & 33 \\
\end{array}$ & $\begin{array}{l}0.4372_{-}^{+} 50 \\
\end{array}$ & $\begin{array}{ll}0.687_{-}^{+} & 8 \\
\end{array}$ & $\begin{array}{l}0.742_{-}^{+} 13 \\
\end{array}$ \\
\hline $0.1565-0.1565$ & $\begin{array}{l}0.3485_{-}^{+} \\
\end{array}$ & $\begin{array}{ll}0.4472_{-}^{+} & 52 \\
\end{array}$ & $0.712_{-}^{+}$ & $\begin{array}{l}0.764_{-}^{+12} \\
\end{array}$ \\
\hline $0.1575-0.1555$ & $\begin{array}{ll}0.3489_{-}^{+} & 31 \\
\end{array}$ & $\begin{array}{l}0.4479_{-}^{+} 55 \\
\end{array}$ & $0.690_{-}^{+}$ & $\begin{array}{l}0.743_{-}^{+} 14 \\
\end{array}$ \\
\hline $0.1570-0.1555$ & $\begin{array}{l}0.3642_{-}^{+30} \\
\end{array}$ & $\begin{array}{l}0.4577_{-}^{+} 52 \\
\end{array}$ & $0.715_{-}^{+}$ & $\begin{array}{l}0.765_{-}^{+} 13 \\
\end{array}$ \\
\hline $0.1565-0.1555$ & $0.3790_{-}^{+28}$ & $\begin{array}{l}0.4677_{-}^{+} 56 \\
\end{array}$ & $0.738_{-}^{+}$ & $\begin{array}{l}0.786_{-}^{+} 11 \\
\end{array}$ \\
\hline $0.1555-0.1555$ & $\begin{array}{l}0.4079_{-}^{+} 27 \\
\end{array}$ & $0.4881_{-}^{+} 47$ & $0.785_{-}^{+}$ & $\begin{array}{l}0.828_{-}^{+} 10 \\
\end{array}$ \\
\hline
\end{tabular}


TABLE IX. Lattice results pseudoscalar and vector meson decay constants at $\kappa_{\text {sea }}=0.156$. For all fits we find $1 \leq \chi^{2} /$ d.o.f $\leq 2$.

\begin{tabular}{|c|c|c|c|c|}
\hline \multicolumn{5}{|c|}{$\kappa_{\text {sea }}=0.156$} \\
\hline \multicolumn{5}{|c|}{ nconfigs $=198$, nboot $=200$, correlated } \\
\hline$\left(t_{\min }, t_{\max }\right)$ & $(9,15)$ & & $(9,15)$ & \\
\hline$\kappa_{1}-\kappa_{2}$ & $\left\langle 0\left|A_{0}^{l}\right| \pi\right\rangle$ & $f_{\pi} / Z_{A}$ & 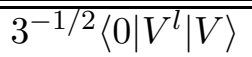 & $1 /\left(Z_{V} f_{\rho}\right)$ \\
\hline $0.1585-0.1585$ & $0.0289_{-}^{+11} 10$ & $0.0985_{-}^{+26}$ & 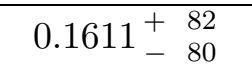 & $0.4758_{-}^{+99} \mid$ \\
\hline $0.1585-0.1580$ & $\begin{array}{l}0.0314_{-}^{+} 12 \\
\end{array}$ & $0.1008_{-}^{+} 25$ & $\begin{array}{c}0.1647_{-}^{+} 78 \\
\end{array}$ & 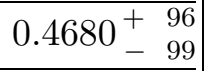 \\
\hline $0.1580-0.1580$ & $\begin{array}{ll}0.0338_{-}^{+12} \\
\end{array}$ & $\begin{array}{l}0.1030_{-}^{+} 27 \\
\end{array}$ & $\begin{array}{l}0.1683_{-}^{+} \\
67\end{array}$ & $\begin{array}{l}0.4609_{-}^{+93} \\
\end{array}$ \\
\hline $0.1585-0.1575$ & $\begin{array}{l}0.0337_{-}^{+} \\
12\end{array}$ & $\begin{array}{l}0.1029_{-}^{+} \\
27 \\
\end{array}$ & $\begin{array}{l}0.1685_{-}^{+} \\
\end{array}$ & $\begin{array}{l}0.4603_{1 \mathrm{e}+02}^{+94} \\
\end{array}$ \\
\hline $0.1580-0.1575$ & $\begin{array}{ll}0.0362_{-}^{+} 13 \\
\end{array}$ & $\begin{array}{c}0.1051_{-}^{+} 28 \\
\end{array}$ & $\begin{array}{c}0.1722_{-}^{+} 73 \\
\end{array}$ & $\begin{array}{r}0.4537_{-}^{+94} \\
\end{array}$ \\
\hline $0.1585-0.1570$ & $\begin{array}{l}0.0361_{-}^{+} 11 \\
\end{array}$ & $\begin{array}{l}0.1049_{-}^{+} 28 \\
\end{array}$ & $\begin{array}{l}0.1725_{-}^{+} 74 \\
\end{array}$ & $\begin{array}{l}0.4526_{-95}^{+96} \\
\end{array}$ \\
\hline $0.1575-0.1575$ & $\begin{array}{ll}0.0386_{-}^{+} 13 \\
\end{array}$ & $0.1073_{-31}^{+} 28$ & $\begin{array}{l}0.1763_{-}^{+} \\
69 \\
\end{array}$ & $\begin{array}{l}0.4472_{-94}^{+90} \\
\end{array}$ \\
\hline $0.1580-0.1570$ & $\begin{array}{ll}0.0385_{-}^{+} 13 \\
\end{array}$ & $\begin{array}{ll}0.1072_{-}^{+} & 28 \\
\end{array}$ & $\begin{array}{ll}0.1763_{-}^{+} & 68 \\
\end{array}$ & $\begin{array}{l}0.4465_{-}^{+91} \\
\end{array}$ \\
\hline $0.1575-0.1570$ & $\begin{array}{l}0.0410_{-}^{+} 14 \\
\end{array}$ & $\begin{array}{l}0.1094_{-}^{+} \quad 29 \\
\end{array}$ & $\begin{array}{l}0.1806_{-}^{+66} \\
\end{array}$ & $\begin{array}{l}0.4405_{-}^{+90} \\
\end{array}$ \\
\hline $0.1585-0.1560$ & $\begin{array}{l}0.0408_{-}^{+} 13 \\
\end{array}$ & $\begin{array}{l}0.1087_{-}^{+} 29 \\
\end{array}$ & $\begin{array}{l}0.1804_{-}^{+} 71 \\
\end{array}$ & $\begin{array}{l}0.4367_{-}^{+} 96 \\
\end{array}$ \\
\hline $0.1570-0.1570$ & $\begin{array}{l}0.0434_{-}^{+} 14 \\
\end{array}$ & 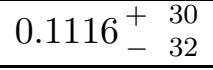 & $\begin{aligned} & 0.1849_{-}^{+} 64 \\
&\end{aligned}$ & $\begin{array}{r}0.4343_{-}^{+} 98 \\
\end{array}$ \\
\hline $0.1580-0.1560$ & $\begin{array}{l}0.0433_{-}^{+} 14 \\
\end{array}$ & 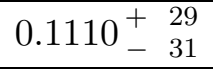 & $\begin{array}{c}0.1846_{-}^{+} \quad 63 \\
\end{array}$ & $\begin{array}{l}0.4317_{-88}^{+} 92 \\
\end{array}$ \\
\hline $0.1575-0.1560$ & $\begin{array}{ll}0.0458_{-}^{+14} \\
\end{array}$ & $\begin{array}{ll}0.1133_{-}^{+} & 29 \\
\end{array}$ & $\begin{array}{l}0.1891_{-}^{+} 63 \\
6\end{array}$ & $\begin{array}{l}0.4266_{-89}^{+90} \\
\end{array}$ \\
\hline $0.1570-0.1560$ & $\begin{array}{ll}0.0483_{-}^{+} 15 \\
\end{array}$ & $0.1156_{-}^{+} \quad 31$ & $\begin{array}{l}0.1937_{-}^{+} \\
62 \\
\end{array}$ & $\begin{array}{l}0.4214_{-}^{+89} \\
\end{array}$ \\
\hline $0.1560-0.1560$ & $\begin{array}{l}0.0534_{-}^{+} 16 \\
\end{array}$ & 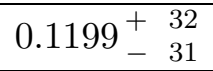 & $\begin{array}{l}0.2030_{-}^{+} 63 \\
\end{array}$ & $\begin{array}{l}0.4103_{-}^{+} 89 \\
\end{array}$ \\
\hline
\end{tabular}


TABLE X. Lattice results pseudoscalar and vector meson decay constants at $\kappa_{\text {sea }}=0.1565$. For all fits we find $1 \leq \chi^{2} /$ d.o.f $\leq 2$.

\begin{tabular}{|c|c|c|c|c|}
\hline \multicolumn{5}{|c|}{$\kappa_{\text {sea }}=0.1565$} \\
\hline \multicolumn{5}{|c|}{ nconfigs $=198$, nboot $=200$, correlated } \\
\hline$\left(t_{\min }, t_{\max }\right)$ & $(9,15)$ & & $(10,15)$ & \\
\hline$\kappa_{1-\kappa_{2}}$ & $\overline{\left\langle\left\langle 0\left|A_{0}^{l}\right| \pi\right\rangle\right.}$ & $f_{\pi} / Z_{A}$ & 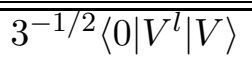 & $\overline{1 /\left(Z_{V} f_{\rho}\right)}$ \\
\hline $0.1580-0.1580$ & $\begin{aligned} & 0.0317_{-}^{+} 19 \\
&\end{aligned}$ & $\begin{array}{l}0.1024_{-}^{+} 50 \\
\end{array}$ & $\begin{array}{l}0.162_{-}^{+} 12 \\
\end{array}$ & $\begin{array}{l}0.481_{-}^{+} 17 \\
\end{array}$ \\
\hline $0.1580-0.1575$ & 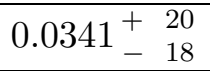 & $\begin{array}{l}0.1047_{-}^{+} 48 \\
\end{array}$ & $\begin{array}{l}0.165_{-}^{+} 11 \\
\end{array}$ & $\begin{array}{l}0.472_{-}^{+} 17 \\
\end{array}$ \\
\hline $0.1575-0.1575$ & $0.0365_{-}^{+} 217$ & $\begin{array}{l}0.1070_{-}^{+} 50 \\
\end{array}$ & 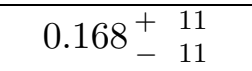 & $\begin{array}{l}0.463_{-}^{+} 17 \\
\end{array}$ \\
\hline $0.1580-0.1570$ & $0.0365_{-}^{+} 217$ & $\begin{array}{l}0.1068_{-}^{+} 50 \\
\end{array}$ & 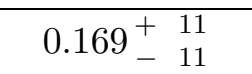 & $\begin{array}{l}0.462_{-}^{+} 16 \\
\end{array}$ \\
\hline $0.1575-0.1570$ & $\begin{array}{l}0.0390_{-}^{+} 22 \\
\end{array}$ & $\begin{array}{l}0.1091_{-}^{+} 49 \\
\end{array}$ & $\begin{array}{l}0.172_{-}^{+} 12 \\
\end{array}$ & $\begin{array}{l}0.454_{-}^{+} 16 \\
\end{array}$ \\
\hline $0.1580-0.1565$ & $\begin{array}{l}0.0389_{-}^{+} \\
\end{array}$ & $\begin{array}{l}0.1087_{-}^{+} 50 \\
\end{array}$ & $\begin{array}{l}0.172_{-}^{+} 12 \\
\end{array}$ & $\begin{array}{l}0.453_{-}^{+} 16 \\
\end{array}$ \\
\hline $0.1570-0.1570$ & $\begin{array}{l}0.0414_{-}^{+} \quad 23 \\
\end{array}$ & $\begin{array}{l}0.1113_{-}^{+} 48 \\
\end{array}$ & $\begin{array}{l}0.176_{-}^{+} 11 \\
\end{array}$ & $\begin{array}{l}0.446_{-}^{+} 15 \\
\end{array}$ \\
\hline $0.1575-0.1565$ & $\begin{array}{l}0.0414_{-}^{+} 23 \\
\end{array}$ & $0.1111_{-}^{+} 48$ & $\begin{array}{l}0.176_{-}^{+11}+11 \\
\end{array}$ & 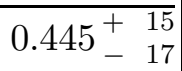 \\
\hline $0.1570-0.1565$ & $\begin{array}{l}0.0439_{-}^{+} 22 \\
\end{array}$ & $\begin{array}{l}0.1133_{-}^{+} \quad 48 \\
\end{array}$ & $\begin{array}{ll}0.180_{-}^{+} & 9 \\
\end{array}$ & $\begin{array}{l}0.438_{-}^{+} 14 \\
\end{array}$ \\
\hline $0.1580-0.1560$ & $\begin{array}{l}0.0412_{-}^{+} 22 \\
-18\end{array}$ & $\begin{array}{l}0.1104_{-}^{+} 50 \\
\end{array}$ & $\begin{array}{l}0.176_{-}^{+12} \\
\end{array}$ & $\begin{array}{l}0.444_{-}^{+} 15 \\
\end{array}$ \\
\hline $0.1565-0.1565$ & $\begin{array}{l}0.0463_{-}^{+} 21 \\
\end{array}$ & $\begin{array}{l}0.1153_{-}^{+} \quad 49 \\
\end{array}$ & $\begin{array}{l}0.184_{-}^{+} \\
\end{array}$ & $\begin{array}{l}0.431_{-}^{+} 14 \\
\end{array}$ \\
\hline $0.1575-0.1560$ & $\begin{array}{l}0.0437_{-}^{+} \\
\end{array}$ & $\begin{array}{l}0.1129_{-}^{+} 49 \\
\end{array}$ & $\begin{array}{l}0.180_{-}^{+} 11 \\
\end{array}$ & $\begin{array}{l}0.437_{-}^{+} 14 \\
\end{array}$ \\
\hline $0.1570-0.1560$ & $\begin{array}{ll}0.0462_{-}^{+} & 22 \\
\end{array}$ & $\begin{array}{l}0.1151_{-}^{+} 48 \\
\end{array}$ & $\begin{array}{l}0.184_{-}^{+} \\
\end{array}$ & $\begin{array}{l}0.430_{-}^{+} 13 \\
\end{array}$ \\
\hline $0.1565-0.1560$ & 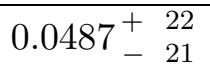 & $0.1172_{-}^{+} 47$ & $\begin{array}{l}0.188_{-}^{+} \\
\end{array}$ & $\begin{array}{l}0.424_{-}^{+} 12 \\
15\end{array}$ \\
\hline $0.1560-0.1560$ & $\begin{array}{l}0.0512_{-}^{+} 25 \\
-21\end{array}$ & $0.1191_{-41}^{+} 47$ & $\begin{array}{ll}0.193_{-}^{+} & 9 \\
\end{array}$ & $\begin{array}{l}0.417_{-}^{+} 12 \\
-14\end{array}$ \\
\hline
\end{tabular}


TABLE XI. Lattice results pseudoscalar and vector meson decay constants at $\kappa_{\text {sea }}=0.157$. For all fits we find $1 \leq \chi^{2} /$ d.o.f $\leq 2$.

\begin{tabular}{|c|c|c|c|c|}
\hline \multicolumn{5}{|c|}{$\kappa_{\text {sea }}=0.157$} \\
\hline \multicolumn{5}{|c|}{ nconfigs $=198$, nboot $=200$, correlated } \\
\hline$\left(t_{\min }, t_{\max }\right)$ & $(10,15)$ & & $(9,15)$ & \\
\hline$\kappa_{1}-\kappa_{2}$ & 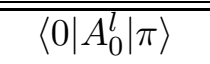 & $f_{\pi} / Z_{A}$ & $\overline{3^{-1 / 2}\left\langle 0\left|V^{l}\right| V\right\rangle}$ & $1 /\left(Z_{V} f_{\rho}\right)$ \\
\hline $0.1575-0.1575$ & $\begin{array}{c}0.0302_{-}^{+} \\
\end{array}$ & $0.0955_{-}^{+27}$ & $\begin{array}{l}0.1491_{-}^{+} 65 \\
6\end{array}$ & $\begin{array}{l}0.4454_{-}^{+} 79 \\
\end{array}$ \\
\hline $0.1575-0.1570$ & $0.0326_{-}^{+} \begin{array}{r}10 \\
\end{array}$ & $\begin{array}{l}0.0979_{-}^{+} 26 \\
\end{array}$ & $\begin{array}{l}0.1538_{-}^{+} \\
64\end{array}$ & $\begin{array}{l}0.4388_{-}^{+} 79 \\
\end{array}$ \\
\hline $0.1570-0.1570$ & $\begin{array}{l}0.0350_{-}^{+} 11 \\
\end{array}$ & $0.1003_{-}^{+} 26$ & $\begin{array}{l}0.1586_{-}^{+} \\
62 \\
\end{array}$ & $\begin{array}{ll}0.4326_{-}^{+} 78 \\
\end{array}$ \\
\hline $0.1575-0.1565$ & $\begin{array}{l}0.0349_{-}^{+} 11 \\
\end{array}$ & $0.1001_{-}^{+} 26$ & $\begin{array}{l}0.1584_{-}^{+} \\
63\end{array}$ & $\begin{array}{l}0.4317_{-}^{+} 75 \\
\end{array}$ \\
\hline $0.1570-0.1565$ & $0.0374_{-}^{+} 12$ & $0.1026_{-}^{+25}$ & $0.1633_{-}^{+} \quad 70$ & $\overline{0.4261_{-}^{+} 75}$ \\
\hline $0.1565-0.1565$ & $0.0398_{-}^{+} 13$ & $0.1050_{-}^{+} 27$ & $0.1680_{-}^{+63}$ & $\begin{array}{l}0.4201_{-}^{+} 73 \\
\end{array}$ \\
\hline $0.1575-0.1555$ & $\begin{array}{l}0.0395_{-}^{+} 12 \\
\end{array}$ & $0.1040_{-}^{+27}$ & $0.1671_{-}^{+} 67$ & $\begin{array}{l}0.4167_{-}^{+} 73 \\
\end{array}$ \\
\hline $0.1570-0.1555$ & $\begin{array}{l}0.0420_{-}^{+} 13 \\
\end{array}$ & $0.1067_{-28}^{+28}$ & $\begin{array}{l}0.1721_{-}^{+} 66 \\
\end{array}$ & $\begin{array}{l}0.4121_{-}^{+}-69 \\
\end{array}$ \\
\hline $0.1565-0.1555$ & $\begin{array}{ll}0.0445_{-}^{+} 14 \\
\end{array}$ & $0.1091_{-28}^{+28}$ & $0.1770_{-}^{+62} 64$ & $0.4071_{-}^{+} 67$ \\
\hline $0.1555-0.1555$ & $0.0493_{-}^{+} 14$ & $0.1133_{-}^{+26}$ & $\begin{array}{l}0.1863_{-}^{+} 63 \\
\end{array}$ & $0.3961_{-}^{+68}$ \\
\hline
\end{tabular}


TABLE XII. Lattice results pseudoscalar and vector meson decay constants at $\kappa_{\text {sea }}=0.1575$. For all fits we find $0.5 \leq \chi^{2} /$ d.o.f $\leq 1$.

\begin{tabular}{|c|c|c|c|c|}
\hline \multicolumn{5}{|c|}{$\kappa_{\text {sea }}=0.1575$} \\
\hline \multicolumn{5}{|c|}{ nconfigs $=198$, nboot $=200$, correlated } \\
\hline$\left(t_{\min }, t_{\max }\right)$ & $(9,15)$ & & $(10,15)$ & \\
\hline$\kappa_{1}-\kappa_{2}$ & $\left\langle 0\left|A_{0}^{l}\right| \pi\right\rangle$ & $\overline{f_{\pi} / Z_{A}}$ & $\overline{3^{-1 / 2}\left\langle 0\left|V^{l}\right| V\right\rangle}$ & $\overline{1 /\left(Z_{V} f_{\rho}\right)}$ \\
\hline $0.1575-0.1575$ & $\begin{array}{ll}0.0251_{-}^{+} & 8 \\
\end{array}$ & $\begin{array}{l}0.0894_{-}^{+26} \\
28\end{array}$ & $0.1286_{-}^{+} 65$ & $\begin{array}{l}0.4443_{-}^{+99} \\
\end{array}$ \\
\hline $0.1575-0.1570$ & $\begin{array}{ll}0.0276_{-}^{+} & 8 \\
\end{array}$ & $\begin{array}{l}0.0924_{-}^{+26} \\
27\end{array}$ & $0.1321_{-60}^{+57}$ & $\begin{array}{l}0.4370_{-}^{+99} \\
\end{array}$ \\
\hline $0.1570-0.1570$ & $\begin{array}{ll}0.0301_{-}^{+} & 8 \\
\end{array}$ & $\begin{array}{l}0.0953_{-}^{+26} \\
\end{array}$ & $0.1361_{-}^{+} 55$ & $\begin{array}{l}0.4305_{-}^{+}+86 \\
\end{array}$ \\
\hline $0.1575-0.1565$ & $\begin{array}{ll}0.0300_{-}^{+} & 8 \\
\end{array}$ & $0.0951_{-26}^{+27}$ & $\begin{array}{l}0.1360_{-}^{+} 53 \\
-57\end{array}$ & $\begin{array}{l}0.4295_{-}^{+}+85 \\
\end{array}$ \\
\hline $0.1570-0.1565$ & $\begin{array}{ll}0.0325_{-}^{+} & 8 \\
\end{array}$ & $0.0979_{-26}^{+24}$ & $0.1403_{-57}^{+53}$ & $\begin{array}{l}0.4239_{-}^{+95} \\
\end{array}$ \\
\hline $0.1565-0.1565$ & $\begin{array}{ll}0.0350_{-}^{+} & 8 \\
\end{array}$ & $0.1004_{-25}^{+23}$ & $0.1448_{-}^{+} 53$ & $\begin{array}{l}0.4180_{-}^{+} 81 \\
\end{array}$ \\
\hline $0.1575-0.1555$ & $\begin{array}{l}0.0347_{-}^{+} \\
10\end{array}$ & $\begin{array}{l}0.0994_{-}^{+27} \\
\end{array}$ & $0.1439_{-}^{+} 51$ & $\overline{0.4141_{-}^{+} 81}$ \\
\hline $0.1570-0.1555$ & $\begin{array}{ll}0.0372_{-}^{+} & 8 \\
\end{array}$ & $\begin{array}{l}0.1021_{-24}^{+23} \\
\end{array}$ & $\begin{array}{l}0.1487_{-}^{+} 52 \\
56\end{array}$ & $\begin{array}{l}0.4099_{-83}^{+90} \\
\end{array}$ \\
\hline $0.1565-0.1555$ & $\begin{array}{lr}0.0396_{-}^{+} & 8 \\
\end{array}$ & $\begin{array}{l}0.1046_{-}^{+23} \\
\end{array}$ & $\begin{array}{l}0.1535_{-}^{+} 52 \\
\end{array}$ & $\begin{array}{l}0.4052_{-85}^{+88} \\
\end{array}$ \\
\hline $0.1555-0.1555$ & $\begin{array}{l}0.0444_{-}^{+} 10 \\
\end{array}$ & $\begin{array}{l}0.1089_{-}^{+21} \\
\end{array}$ & $\begin{array}{l}0.1629_{-}^{+} 47 \\
\end{array}$ & $\begin{array}{l}0.3948_{-80}^{+83} \\
\end{array}$ \\
\hline
\end{tabular}

TABLE XIII. Fit results for pseudoscalar, vector, nucleon and delta particles (in lattice units) from "symmetric" fits.

\begin{tabular}{|c|cccc|}
\hline \hline particle & $a$ & $b$ & & $\chi^{2} /$ d.o.f \\
\hline $\mathrm{PS}$ & $-12.407(380)$ & $1.9666(540)$ & $e$ & $1.2 / 2$ \\
\hline \hline particle & $m^{\text {crit }}$ & $c$ & 0 & $\chi^{2} /$ d.o.f \\
\hline $\mathrm{V}$ & $0.3300(90)$ & $4.070(250)$ & $-33.28(34.1)$ & $1.5 / 2$ \\
$\mathrm{~V}$ & $0.2928(410)$ & $6.44(2.40)$ & 0 & $0.27 / 1$ \\
\hline $\mathrm{N}$ & $0.5012(190)$ & $6.960(460)$ & $-67.99(69.1)$ & $2.7 / 2$ \\
$\mathrm{~N}$ & $0.4246(750)$ & $11.84(4.8)$ & 0 & $1.5 / 1$ \\
\hline$\Delta$ & $0.5851(240)$ & $6.482(670)$ & 0 & $6 / 2$ \\
$\Delta$ & $0.444(110)$ & $15.16(6.3)$ & $-118.9(82.0)$ & $3.5 / 1$ \\
\hline$f_{\pi}$ & $0.0496(34)$ & $0.888(101)$ & $0.58(11.15)$ & $1.3 / 2$ \\
$f_{\pi}$ & $0.0423(137)$ & $1.354(778)$ & $-7.45(31.44)$ & $0.89 / 1$ \\
\hline $1 / f_{\rho}$ & $0.302(110)$ & $-0.372(290)$ & & $0.37 / 2$ \\
$1 / f_{\rho}$ & $0.294(43)$ & $0.170(2.204)$ & & $0.31 / 1$ \\
\hline \hline
\end{tabular}


TABLE XIV. Values of the coupling constant. We do not take into account the light-quark dependence of the plaquette in the determination of the strong coupling constant.

\begin{tabular}{|c|c|ccc|}
\hline \hline & $S_{\square}$ & $\alpha_{V}(3.41 / a)$ & $\alpha_{\overline{M S}}(\pi / a)$ & $\alpha_{\overline{M S}}(1 / a)$ \\
\hline \hline$\beta=5.6, N_{F}=2$ & $(0.43012,0.42927,0.42837,0.42749)$ & 0.167 & 0.150 & 0.215 \\
\hline$\beta=6.0, N_{F}=0$ & 0.406318 & 0.152 & 0.138 & 0.205 \\
\hline \hline
\end{tabular}

TABLE XV. Fit results for the masses of pseudoscalar and vector particles in the strange sector, according to eqs. 15, 17 and 18

\begin{tabular}{|ccccc|}
\hline \hline PS(linear fit) & & $a^{\prime}$ & $b^{\prime}$ & $\chi^{2} /$ d.o.f \\
\hline & - & $3.93(12)$ & $1,01(11)$ & $26 / 31$ \\
\hline \hline V(linear fit) & $m^{\prime \text { crit }}$ & $c^{\prime}$ & $d^{\prime}$ & $\chi^{2} /$ d.o.f \\
\hline & $0.3300(93)$ & $4.07(25)$ & $0.948(31)$ & $13 / 31$ \\
\hline \hline V(quadr. fit) & $m^{\prime \text { crit }}$ & $c^{\prime}$ & $d^{\prime}$ & $\chi^{2} /$ d.o.f \\
\hline & $0.2928(412)$ & $6.44(2.4)$ & $0.908(145)$ & $3 / 29$ \\
\hline & $e^{\prime}$ & $f^{\prime}$ & $g^{\prime}$ & \\
\hline \hline & $-33.28(34.3)$ & $-2.19(4.79)$ & $2.25(2.03)$ & \\
\hline \hline
\end{tabular}

TABLE XVI. Collection of results for $\kappa^{\text {strange }}$.

\begin{tabular}{|ccc|}
\hline \hline particle & $\kappa_{\text {lin }}^{\text {strange }}$ & $\kappa_{\text {quad }}^{\text {strange }}$ \\
\hline$K$ & $0.15654(11)$ & $0.15694(42)$ \\
\hline$K^{*}$ & $0.15561(14)$ & $0.15590(57)$ \\
\hline$\phi$ & $0.15563(14)$ & $0.15598(50)$ \\
\hline \hline
\end{tabular}


TABLE XVII. Lattice results in the strange quark sector.

\begin{tabular}{|ccc|}
\hline \hline observable & lin fit to $m_{\rho}$ & quad fit to $m_{\rho}$ \\
\hline$m_{K}(\phi)$ & $0.259(6)$ & $0.241(25)$ \\
$m_{\phi}(\phi)$ & $0.443(12)$ & $0.395(54)$ \\
$m_{K^{*}}(\phi)$ & $0.388(11)$ & $0.345(47)$ \\
\hline$m_{K}\left(K^{*}\right)$ & $0.259(6)$ & $0.245(28)$ \\
$m_{\phi}\left(K^{*}\right)$ & $0.443(12)$ & $0.398(56)$ \\
$m_{K^{*}}\left(K^{*}\right)$ & $0.288(11)$ & $0.346(48)$ \\
\hline$m_{K}(K)$ & $0.215(6)$ & $0.192(26)$ \\
$m_{\phi}(K)$ & $0.407(13)$ & $0.356(53)$ \\
$m_{K^{*}}(K)$ & $0.371(11)$ & $0.326(47)$ \\
\hline$f_{K}(\phi)$ & $0.0633(33)$ & $0.0615(38)$ \\
$1 / f_{\phi}(\phi)$ & $0.2742(93)$ & $0.2777(97)$ \\
\hline$f_{K}\left(K^{*}\right)$ & $0.0633(33)$ & $0.0619(38)$ \\
$1 / f_{\phi}\left(K^{*}\right)$ & $0.2741(93)$ & $0.2769(104)$ \\
\hline$f_{K}(K)$ & $0.0591(33)$ & $0.0572(34)$ \\
$1 / f_{\phi}(K)$ & $0.2832(97)$ & $0.2871(105)$ \\
\hline \hline
\end{tabular}

TABLE XVIII. Values of the inverse lattice spacing obtained from different observables (at the light quark mass). The lattice value of $m_{N}$ results from a quadratic extrapolation, the value of $f_{\pi}$ stems from a linear extrapolation.

\begin{tabular}{|ccc|}
\hline \hline observable & $m\left(\kappa_{\text {sea }}^{\text {light }}\right)$ & $a^{-1}$ \\
\hline$m_{\rho}$ (linear fit) & $0.334(9)$ & $2.30(6)$ \\
\hline$m_{\rho}$ (quadratic fit) & $0.297(41)$ & $2.58(37)$ \\
\hline$m_{N}$ (rho linear) & $0.435(72)$ & $2.16(40)$ \\
\hline$f_{\pi}$ (rho linear) & $0.0505(34)$ & $2.62(18)$ \\
\hline \hline
\end{tabular}

TABLE XIX. Physical results in the light quark sector.

\begin{tabular}{|cccc|}
\hline \hline$m_{N}[\mathrm{GeV}]$ & $m_{\Delta}[\mathrm{GeV}]$ & $f_{\pi}[\mathrm{GeV}]$ & $1 / f_{\rho}$ \\
\hline linear vector & & & $0.302(11)$ \\
\hline $1.00(16)$ & $1.05(23)$ & $0.116(8)$ & \\
\hline quadr. vector & & & $0.302(11)$ \\
\hline $1.12(25)$ & $1.17(28)$ & $0.130(21)$ & \\
\hline \hline
\end{tabular}


TABLE XX. Physical results for masses in the strange quark sector.

\begin{tabular}{|cccccc}
\hline \hline$m_{K}\left(K^{*}\right)[\mathrm{GeV}]$ & $m_{K}(\phi)[\mathrm{GeV}]$ & $m_{K^{*}}(\mathrm{~K})[\mathrm{GeV}]$ & $m_{K^{*}}(\phi)[\mathrm{GeV}]$ & $m_{\phi}(\mathrm{K})[\mathrm{GeV}]$ & $m_{\phi}\left(K^{*}\right)[\mathrm{GeV}]$ \\
\hline linear vector & \multicolumn{5}{c}{} \\
\hline $0.596(9)$ & $0.595(9)$ & $0.853(3)$ & 0.894 & $0.937(6)$ & 1.020 \\
\hline quadr. vector & & & & \\
\hline $0.633(47)$ & $0.623(44)$ & $0.842(12)$ & $0.890(4)$ & $0.918(24)$ & $1.029(9)$ \\
\hline \hline
\end{tabular}

TABLE XXI. Physical results for decay constants in the strange quark sector.

\begin{tabular}{|lccccc|}
\hline \hline$f_{K}(\phi)[G e V]$ & $f_{K}\left(K^{*}\right)[G e V]$ & $f_{K}(K)[G e V]$ & $1 / f_{\phi}(\phi)$ & $1 / f_{\phi}\left(K^{*}\right)$ & $1 / f_{\phi}(K)$ \\
\hline linear vector & & & & & \\
\hline $0.1456(71)$ & $0.1457(71)$ & $0.1360(72)$ & $0.2742(93)$ & $0.2741(93)$ & $0.2832(97)$ \\
\hline \hline
\end{tabular}

TABLE XXII. Physical results in the quenched sector. Fits to nucleon and $\Delta$ are quadratic, all other fits are linear. To set $\kappa^{\text {strange }}$ we used the Kaon mass for $f_{K}$ and $m_{K^{*}}$, and the $\phi$ mass for $1 / f_{\phi}, m_{K}$ and $J$.

\begin{tabular}{|lcccc|}
\hline \hline$m_{N}[\mathrm{GeV}]$ & $m_{\Delta}[\mathrm{GeV}]$ & $f_{\pi}[\mathrm{GeV}]$ & $1 / f_{\rho}$ & \\
\hline $1.061(67)$ & $1.301(63)$ & $0.1325(48)$ & $0.3271(52)$ & \\
\hline$m_{K}[\mathrm{GeV}]$ & $m_{K^{*}}[\mathrm{GeV}]$ & $f_{K}[\mathrm{GeV}]$ & $1 / f_{\phi}$ & $J$ \\
\hline $0.5590(64)$ & $0.8647(23)$ & $0.1518(46)$ & $0.2982(47)$ & $0.38(1)$ \\
\hline \hline
\end{tabular}




\section{FIGURES}
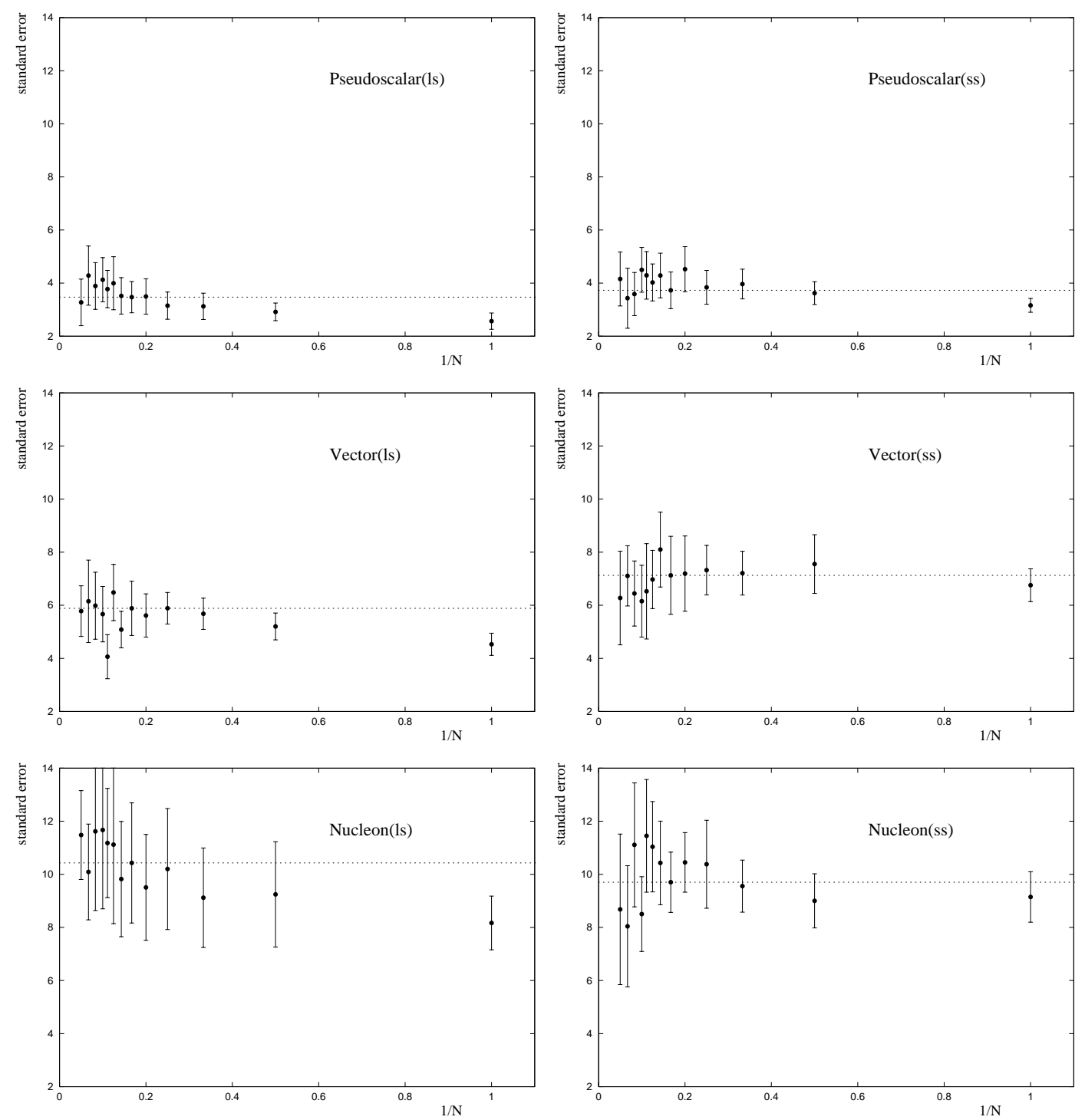

FIG. 1. The standard error of the masses of pseudoscalar, vector and nucleon as a function of the inverse block size 


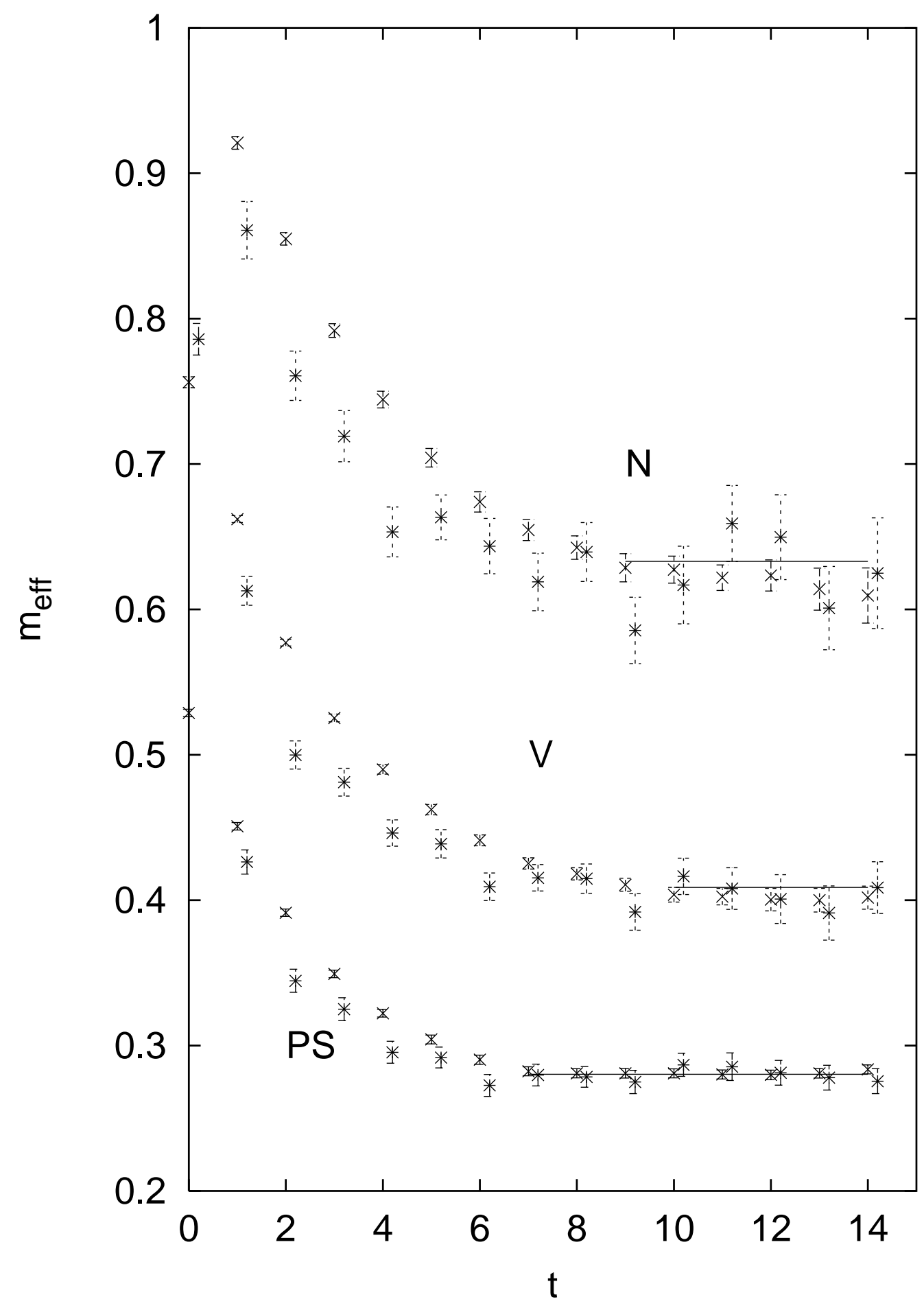

FIG. 2. Effective masses at $\kappa_{\text {sea }}=0.1575 . *$ are smeared-smeared and $\times$ are smeared-local data. The results of our fits to the smeared-smeared correlators (not to the effective masses!) are indicated by solid lines. 


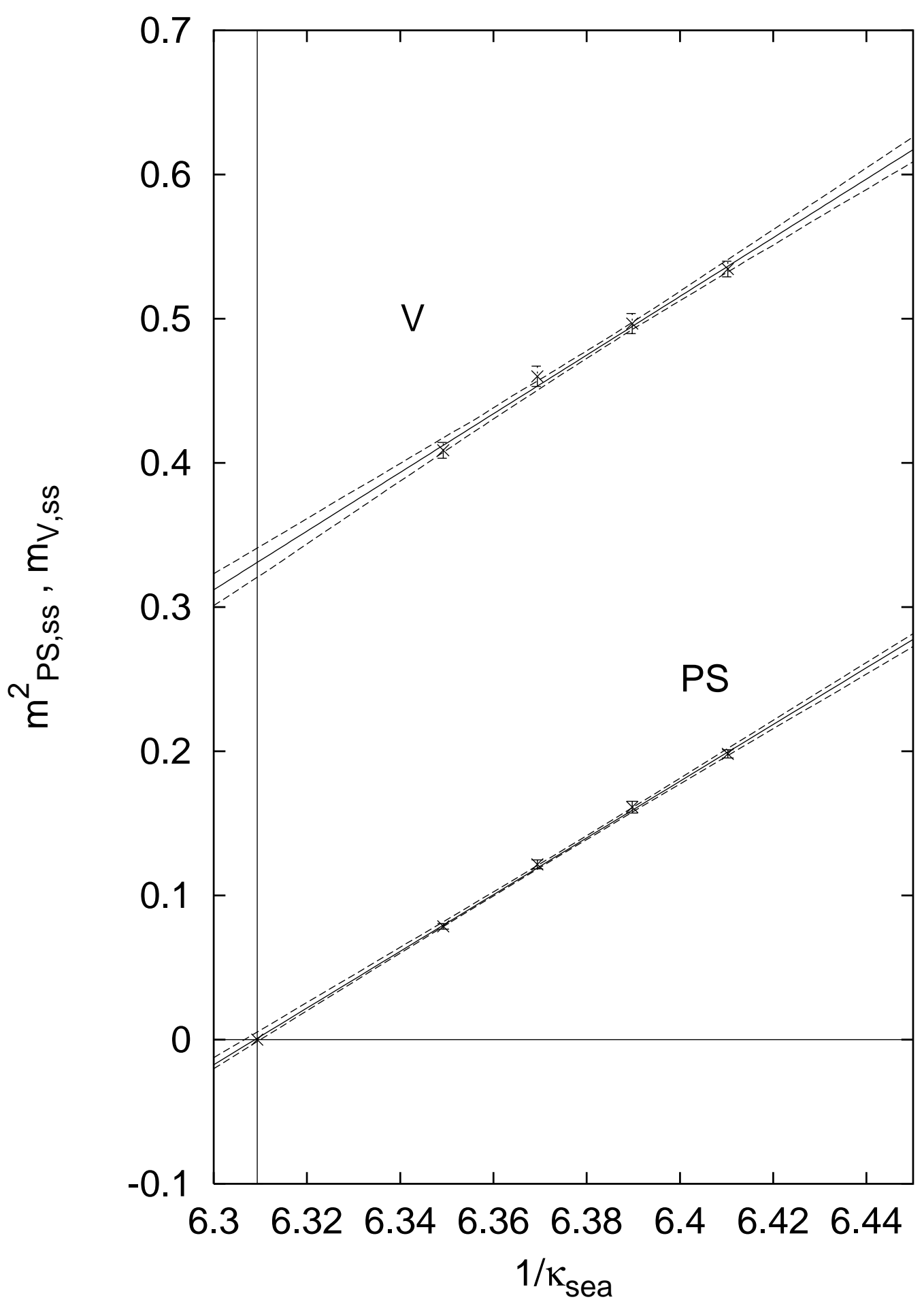

FIG. 3. $m_{\mathrm{PS}, \mathrm{ss}}^{2}$ and $m_{\mathrm{V}, \mathrm{ss}}$ as a function of $\frac{1}{\kappa_{\mathrm{sea}}}$ (in lattice units). 


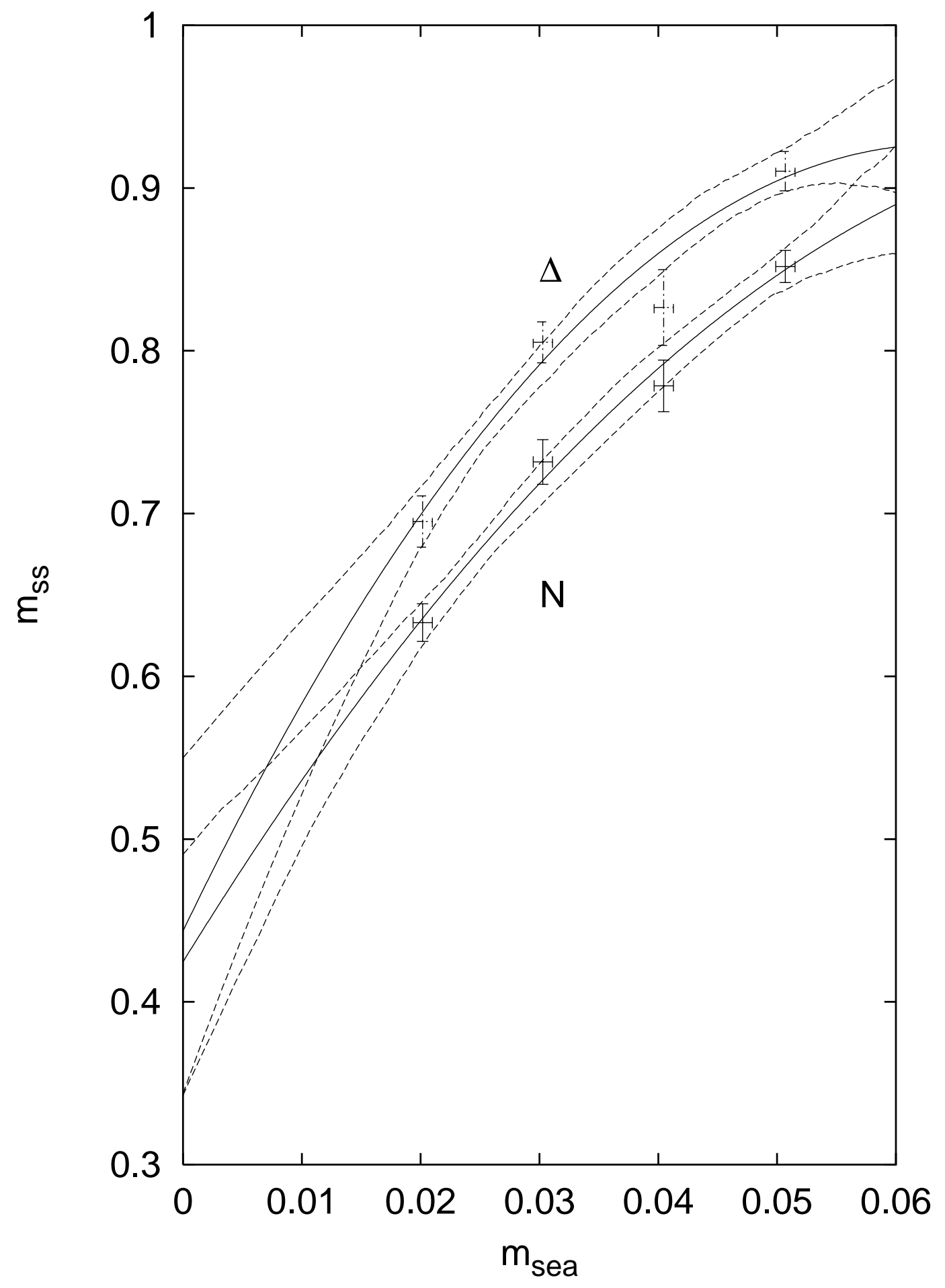

FIG. 4. $m_{N}$ and $m_{\Delta}$ as a function of $m_{\text {sea }}$ (in lattice units). 

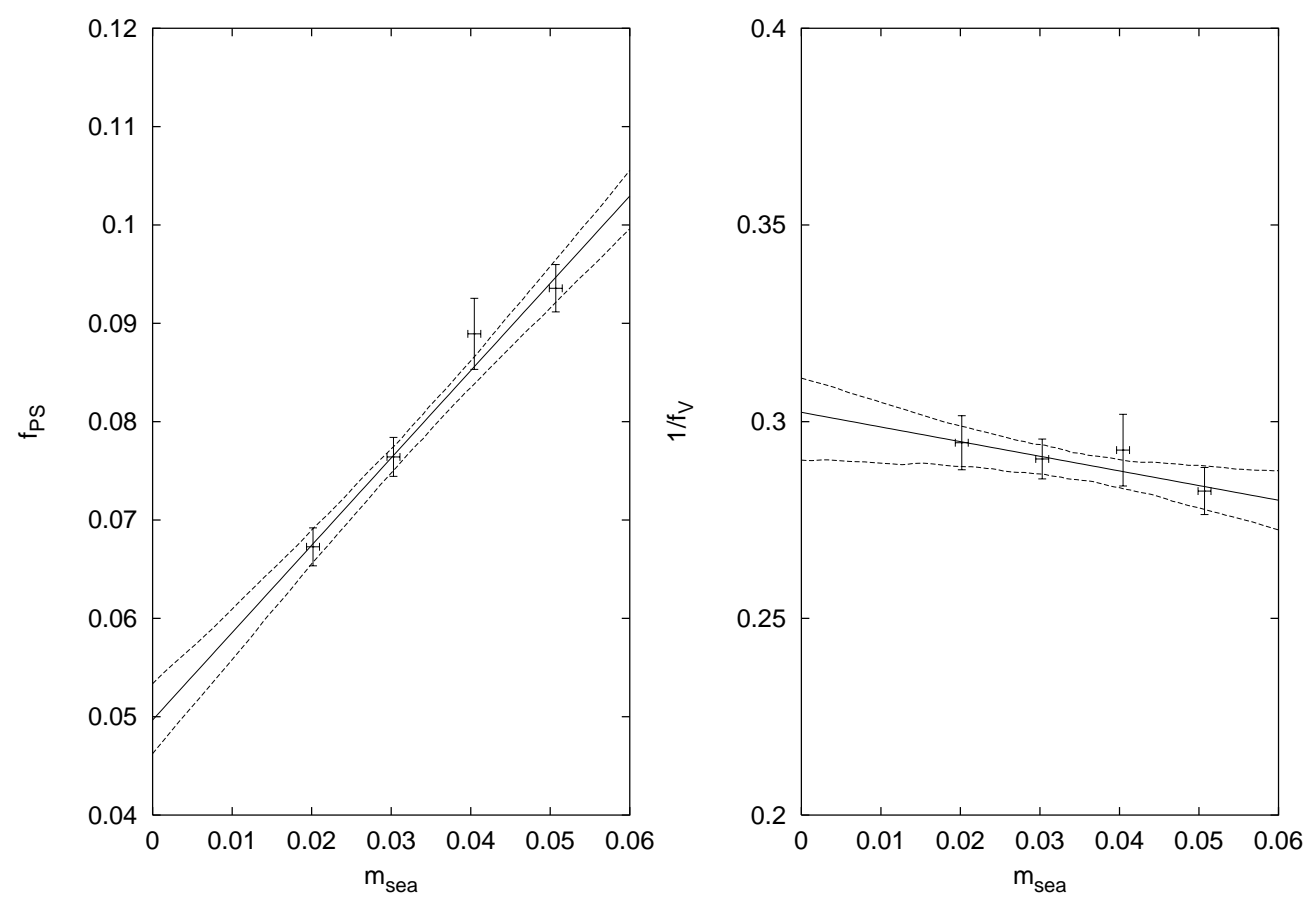

FIG. 5. Linear chiral extrapolations of $f_{P S}$ and $1 / f_{V}$
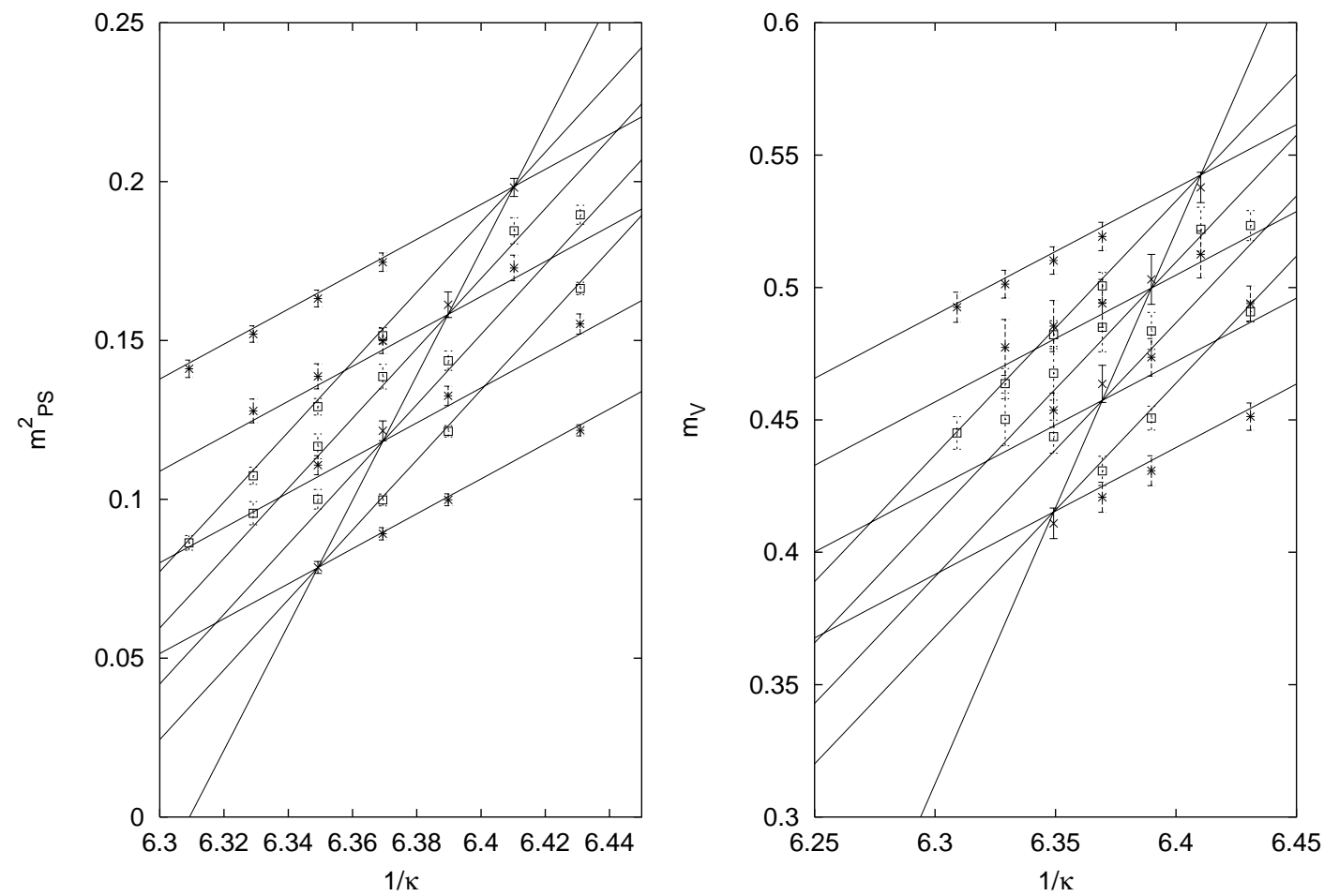

FIG. 6. Simultaneous fit of all pseudoscalar-data and vector-data to eq. 15. Symbols: $*=m_{\mathrm{ss}} ; \diamond=m_{\mathrm{sv}} ; \square=m_{\mathrm{vv}}$. 


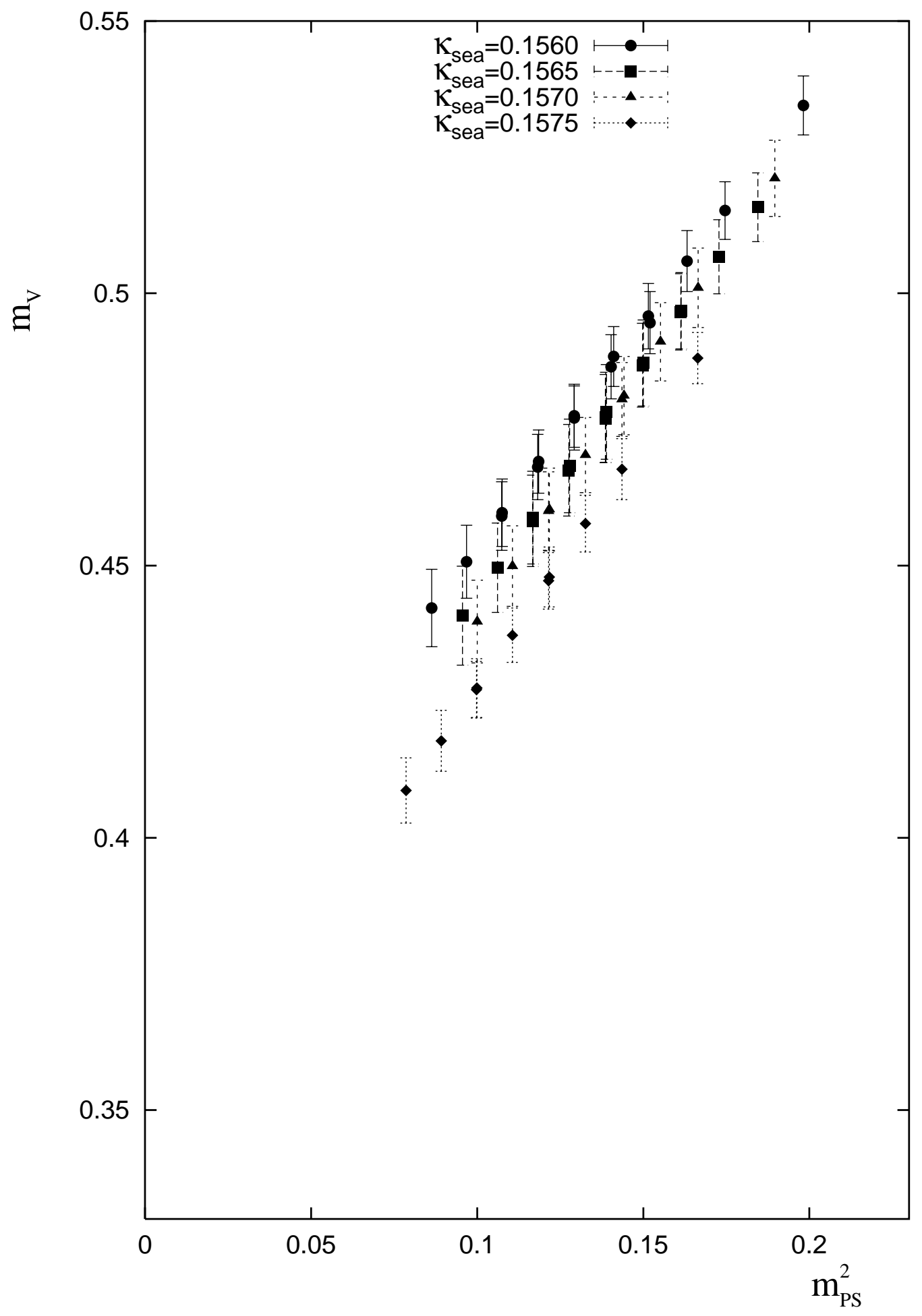

FIG. 7. Mass of the vector meson as a function of $m_{\pi}^{2}$. 


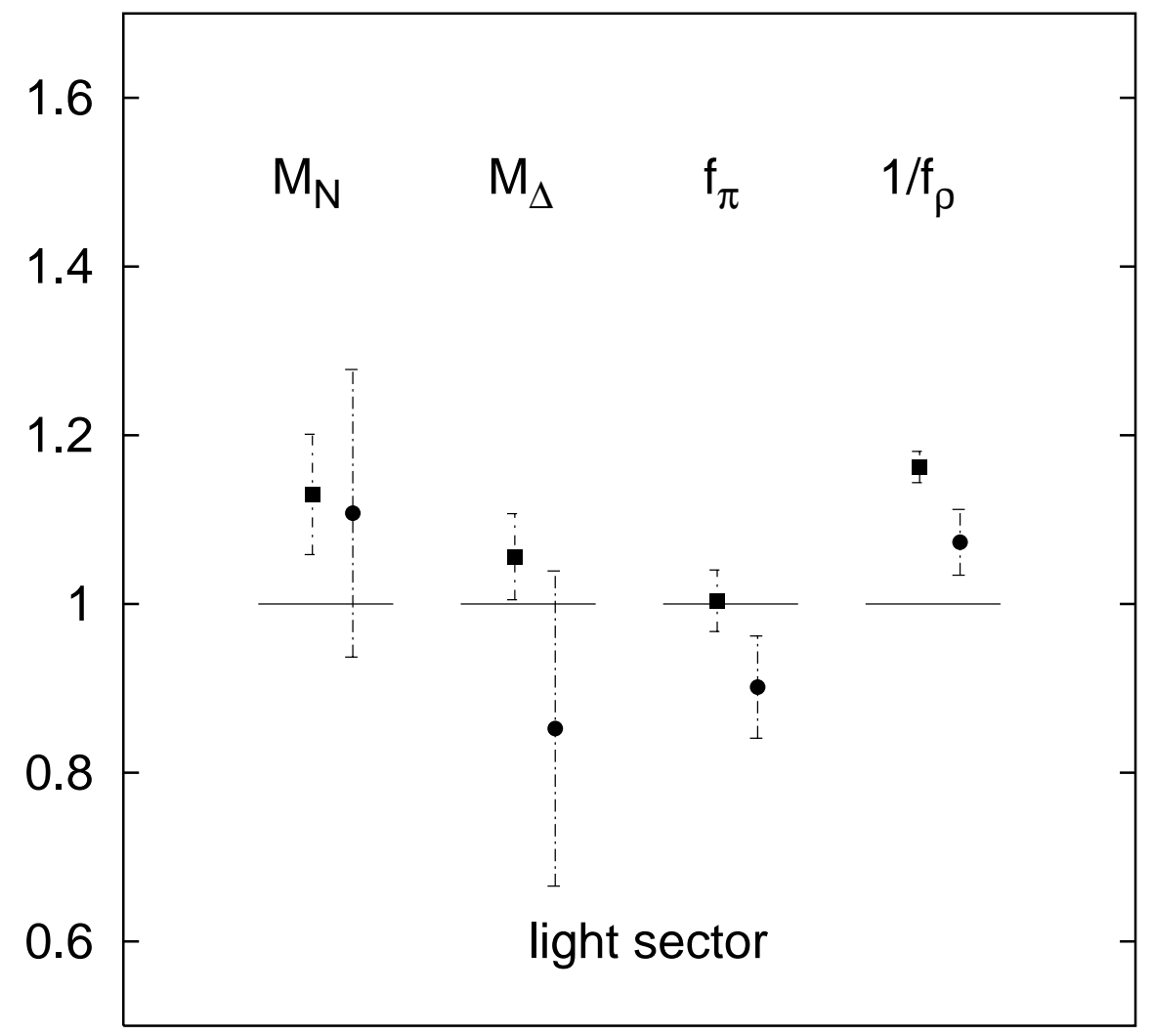

FIG. 8. Comparison of $N_{F}=2$ (circles) and quenched results (squares) in the light sector with experiment. The data is normalized to its experimental values, namely $M_{N}=938 \mathrm{MeV}$, $M_{\Delta}=1232 \mathrm{MeV}, f_{\pi}=132 \mathrm{MeV}, 1 / f_{\rho}=0.199 \sqrt{2}$. To set the scale we used the linear fit to the vector meson trajectory. 


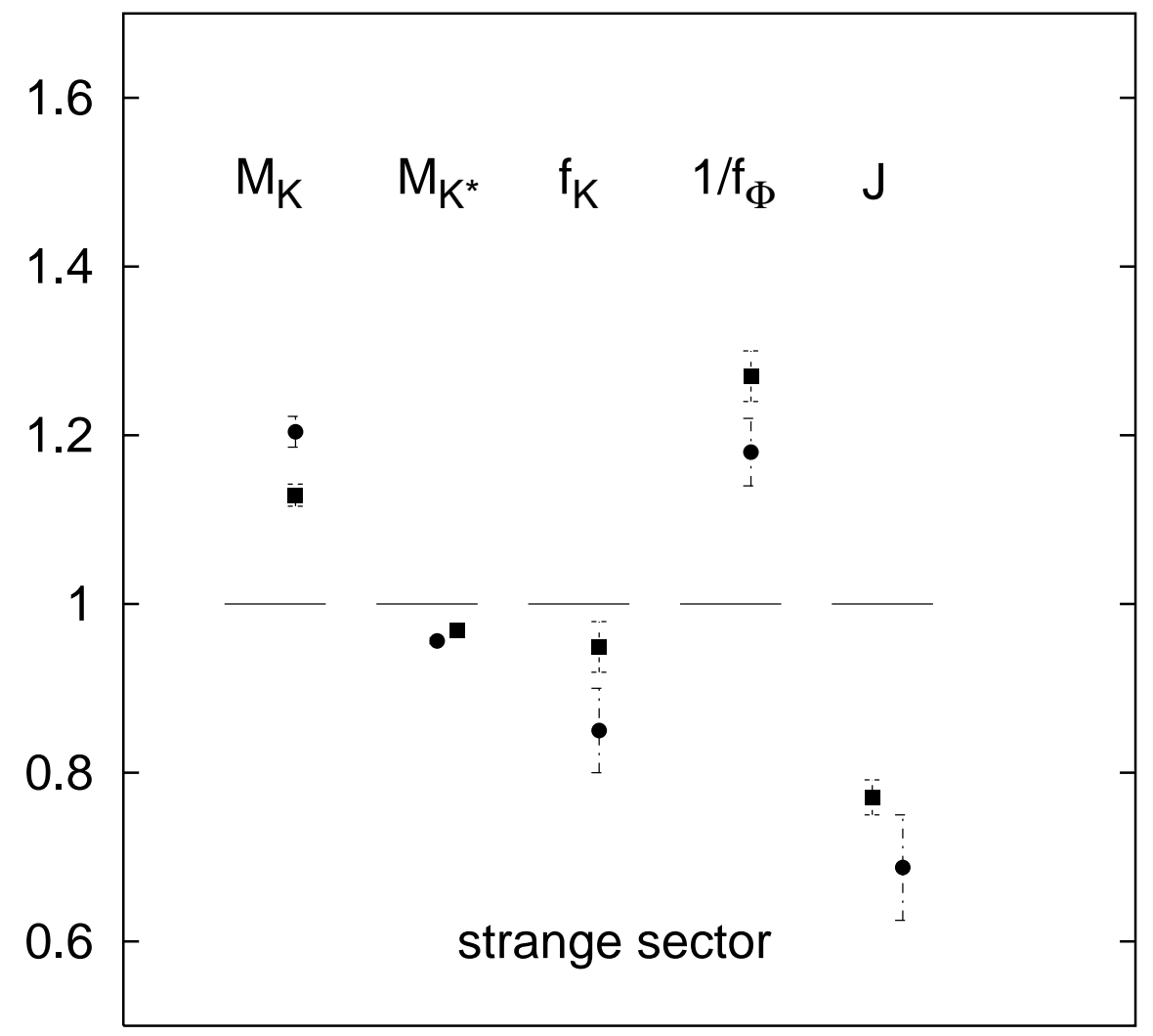

FIG. 9. Comparison of $N_{F}=2$ (circles) and quenched results (squares) in the strange sector with experiment. The data is normalized to its experimental values, namely $M_{K}=495 \mathrm{MeV}$, $M_{K^{*}}=892 \mathrm{MeV}, f_{K}=160 \mathrm{MeV}, 1 / f_{\Phi}=0.234$ and $J=0.48$. To set the scale we used the linear fit to the vector meson trajectory. 